\title{
Die Funktion des Verfahrensrechts in privatisierten Be- reichen - Verfahren als Gegenstand der Regulierung nach Verantwortungsteilung -
}

\author{
Martin Burgi
}

\section{Inhaltsübersicht}

A. Stand der Verwaltungsreformdebatte und Aufgabenstellung 156

I. Die Stunde der Verwaltungsrechtsdogmatik 156

II. Verfahren und Privatisierung 159

B. Grobstrukturierung der »privatisierten Bereiche« 161

I. "Privatisierte Bereiche« als Produkt von Verantwortungsteilungen 162

II. (Regulierte) Verwaltungshilfe (nach funktionaler Privatisierung) 163

III. Regulierte Selbstregulierung 164

1. Begriff und Spektrum 164

2. Unterscheidungskriterium »Entscheidungsbezug" 168

IV. Ausgeschiedene Bereiche 170

C. Vergewisserung: Funktionen des Verwaltungsverfahren(-srechts) bei staatlicher Erfüllungsverantwortung

I. Bewirkungsfunktion und Schutzfunktion 171

II. Teil-Funktionen und einzelne Verfahrenselemente 172

1. Distanz 172

2. Sachaufklärung und Betroffenenbeteiligung 172

3. Transparenz, Beteiligtenanhörung und Begründung 173

III. Verfabren und Organisation 173

IV. Verfassungsrechtliche Impulse und Mindeststandards 174

D. Funktionsanalyse nach Privatisierung: Funktionale Verfahrens-
Äquivalenz als Notwendigkeit und Option

I. Fragestellung 174

II. Verwaltungshilfe: Private Vorbereitung staatlicher Entscheidungen $\quad 175$

III. Regulierte Selbstregulierung 177

1. Bezug zu staatlichen Entscheidungen (Identität des materiellen Maßstabes) 177

2. Fehlender Bezug zu staatlichen Entscheidungen 178

E. Verfahren als Gegenstand der Regulierung: Schaffung funktionaler
Äquivalente

$\begin{array}{ll}\text { I. Strukturschaffungspflicht statt unmittelbare (verfassungs-) } & \\ \text { verfahrensgesetzliche Bindung } & 179\end{array}$

II. Verfahrensrechtsverhältnisse nach Privatisierung 181

1. Staat-Dritte 182 
2. Staat - private Träger (ggf. mit privater Implementationsinstanz)

3. Private Träger (ggf. mit privater Implementationsinstanz) Dritte

III. Einzelne Verfahrenselemente 184

1. Distanz 184

2. Sachaufklärung und Betroffenenbeteiligung 186

3. Transparenz, Beteiligtenanhörung und Begründung 187

IV. Standorte normativer Verfahrens-Regulierung 188

1. Bei ausschließlich normativer Regulierung 189

2. Bei auch administrativer Regulierung 189

F. Schluss

Der folgende Beitrag ist im Überschneidungsbereich zweier Problemkreise angesiedelt, wobei sich der Überschneidungsbereich als gleichermaßen schmal wie kompliziert erweist, da das Verfahrensrecht in seiner ganzen Vielfalt betroffen ist. Um die verfahrensrechtlichen Herausforderungen in diesem Bereich bewältigen zu können, muss der Blick weit in den Problemkreis der Privatisierung hinüber gerichtet werden.

\section{A. Stand der Verwaltungsreformdebatte und Aufgabenstellung}

\section{Die Stunde der Verwaltungsrechtsdogmatik}

Im Themenfeld »Der Verfahrensgedanke nach Privatisierung « lautet das Gebot der Stunde dahin, rechts- wie verwaltungswissenschaftliche Theorie, administrative wie judikative ${ }^{1}$ Praxis und Rechtspolitik zusammenzuführen und eine dogmatische Basis für die Bewältigung der verfahrensmäßigen Herausforderungen von »Public Private Partnership«, »Verantwortungsteilung « bzw. »regulierter Selbstregulierung« zu schaffen. Die Uneinheitlichkeit der Terminologie offenbart Vorsprung und Dominanz der Theorie, entfaltet in verschiedenen Bänden des DFG-Reformzyklus zur Reform des Verwaltungsrechts (v. a.: »Öffentliches Recht und Privatrecht als wechselseitige Auffang-

1 Erstaunlicherweise haben die nunmehr seit vielen Jahren praktizierten und vom Schriftum begleiteten Privatisierungen nicht für ein entsprechend gestiegenes Aufkommen im Bereich der Dritten Gewalt gesorgt; die Zahl der einschlägigen Gerichtsentscheidungen ist zu niedrig, als dass man von einer »Privatisierungs-Rechtsprechung« sprechen könnte. 
ordnungen $\aleph^{2}$ und »Verwaltungsorganisationsrecht als Steuerungsressource $\ll^{3}$ ), flankiert von drei spezielleren Tagungsbänden (zur »Verfahrens-

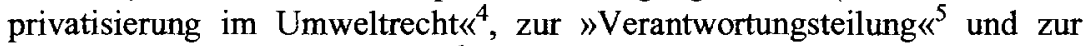
»regulierten Selbstregulierung $\aleph^{6}$ ) sowie von bilanzierend-forcierenden Einzelbeiträgen ${ }^{7}$. Dabei konnte aufgezeigt werden, dass die Einbeziehung Privater in die Gemeinwohlverwirklichung überhaupt verfahrensmäßige Dimensionen hat und dass Bedarf für ein »Privatverfahrens- bzw. Privatorganisationsrecht« besteht; ferner wurden erste konzeptionelle Vorstellungen über dessen Ausgestaltung formuliert ${ }^{8}$. Neben diesen primär theoretischen Arbeiten widmeten sich andere Autoren mit einem bereits dogmatischen Ansatz Teilausschnitten der Problematik auf der konkreteren Ebene der verfassungs-

2 Erschienen 1996 unter der Herausgeberschaft von Hoffmann-Riem/Schmidt-ABmann; aus diesem Band sind für das vorliegende Thema insbesondere relevant die Beiträge von Schmidt-Aßmann, Offentliches Recht und Privatrecht: Ihre Funktionen als wechselseitige Auffangordnungen, S. 7 ff., Trute, Verzahnungen von öffentlichem und privatem Recht, S. 167 ff., und Hoffmann-Riem, Öffentliches Recht und Privatrecht als wechselseitige Auffangordnungen - Systematisierung und Entwicklungsperspektiven, S. $261 \mathrm{ff}$.

3 Im Jahre 1997 erschienen unter der Herausgeberschaft von Schmidt-Aßmann/HoffmannRiem; vgl. dort v.a. Schmidt-Aßmann, Verwaltungsorganisationsrecht als Steuerungsressource, S. 9 ff., Trute, Funktionen der Organisation und ihre Abbildung im Recht, S. 249 ff, Wahl, Privatorganisationsrecht als Steuerungsinstrument bei der Wahmehmung öffentlicher Aufgaben, S. $301 \mathrm{ff}$., und Hoffmann-Riem, Organisationsrecht als Steuerungsressource. Perspektiven, S. $355 \mathrm{ff}$.

41996 erschienen unter der Herausgeberschaft von Hoffmann-Riem/Schneider. Neben dem textgleich auch in DVBl. 1996, S. 225 ff., erschienenen Eröffnungsvortrag von HoffmannRiem, Verfahrensprivatisierung als Modernisierung (der im folgenden hiernach zitiert wird), werden darin zahlreiche Anwendungsbeispiele aus verschiedenen Referenzgebieten vorgestellt.

5 Der vollständige Titel des von Schuppert im Jahre 1999 herausgegebenen Bandes lautet: »Jenseits von Privatisierung und schlankem Staat. Verantwortungsteilung als Schlüsselbegriff eines sich verändernden Verhältnisses von öffentlichem und privatem Sektor «; vgl. v.a. Trute, Verantwortungsteilung als Schlūsselbegriff eines sich verändernden Verhältnisses von öffentlichem und privatem Sektor, S. 13 ff., Voßkuhle, Gesetzgeberische Regelungskategorien der Verantwortungsteilung zwischen öffentlichem und privatem Sektor, S. $47 \mathrm{ff}$., Laskowski, Durch Verantwortungsstrukturen im Umweltrecht und Umweltpolitik, S. 93 ff., und Bauer, Zur notwendigen Entwicklung eines Verwaltungskooperationsrechts, S. $249 \mathrm{ff}$.

6 In dem 2001 als Beiheft 4 der Zeitschrift Die Verwaltung (DV), erschienenen Band »Regulierte Selbstregulierung als Steuerungskonzept des Gewäbrleistungsstaates« sind die Ergebnisse eines Symposiums aus Anlass des 60. Geburtstages von Hoffmann-Riem zusammengefasst; vgl. v.a. Voßkuhle, »Regulierte Selbstregulierung« - zur Karriere eines Schlüsselbegriffs, S. $197 \mathrm{ff}$., Schuppert, Das Konzept der regulierten Selbstregulierung als Bestandteil einer als Regelungswissenschaft verstandenen Rechtswissenschaft, S. 201 ff., Schmidt$A \beta m a n n$, Regulierte Selbstregulierung als Element verwaltungsrechtlicher Systembildung, S. $253 \mathrm{ff}$.

7 Vgl. an dieser Stelle nur Röhl, Verwaltungsverantwortung als dogmatischer Begriff, DV 1999, Beiheft 2, S. 33 ff.; Voßkuhle, "Schlüsselbegriffe « in der Verwaltungsrechtsreform eine kritische Bestandsaufnahme -, VerwArch 92 (2001), S. 184.

8 Von Ritter, Organisationswandel durch Expertifizierung und Privatisierung im Ordnungsund Planungsrecht, in: Hoffmann-Riem/Schmidt-ABmann, Verwaltungsorganisationsrecht (Fn. 3), S. 207 (235 ff.). 
rechtlichen Anforderungen ${ }^{9}$ bzw. der einfachrechtlichen und verwaltungspraktischen Ausformung in einzelnen Referenzgebieten bzw. mit Blick auf bestimmte private Beiträge bereichsübergreifenden Charakters (wie die Sachverständigkeit) ${ }^{10}$. Auf der rechtspolitischen Ebene (bezogen auf das allgemeine Verwaltungsrecht) ist nun dadurch Bewegung entstanden, dass das Bundesinnenministerium im Zuge seines Reformprojektes »Moderner Staat moderne Verwaltung« über die Schaffung eines Rechtsrahmens für »Public Private Partnerships « nachdenkt ${ }^{11}$ und nachdenken lässt ${ }^{12}$.

Der Zeitpunkt, die verschiedenen systembildenden Kräfte zu bündeln, und die klassischen dogmatischen Anliegen »Entlastung der Rechtspraxis, Systembildung, Rationalisierungs- und Orientierungshilfe ${ }^{13}$, zu verwirklichen, ist also gut gewählt. Der »Beruf unserer Zeit« in Fragen der Verantwortungsteilung liegt $\mathrm{m}$. E. darin, einerseits die bestehenden rechtspraktischen und dogmatischen Ansätze theoretisch zu spiegeln und andererseits die theoretischen Konzepte in vergleichsweise »einfache, juristische Grundformen« (Fritz Flei$n e r)^{14} \mathrm{zu}$ überführen, um damit das für den täglichen Einsatz erforderliche $\mathrm{MaB}$ an Verfestigung und Aussagekraft zu erreichen. Das berechtigt m. E. auch dazu, den thematischen Rahmen an einigen Stellen sachgerecht zu verengen.

9 Burgi, Privat vorbereitete Verwaltungsentscheidungen und staatliche Strukturschaffungspflicht. Verwaltungsverfassungsrecht im Kooperationsspektrum zwischen Staat und Gesellschaft, DV 33 (2000), S. 183.

10 Vgl, neben einzelnen Beiträgen in den in Fn. 3 bzw. 4 genannten Sammelbänden und den zu B II und III zitierten Arbeiten die sich mit dem Sachverständigenwesen befassenden Untersuchungen von Seidel, Privater Sachverstand und staatliche Garantenstellung im Verwaltungsrecht, 2000, S. 123 ff., 1955 ff.; Scholz, Für ein Sachverständigengesetz!, ZG 2000, $231 \mathrm{ff}$.

11 Die diesbezüglichen Vorstellungen sind vorgestellt im Internet unter www.staat-modern.de/ projekte; vgl. auch Schmitz, Modemer Staat - Modernes Verwaltungsverfahrensrecht, NVwZ 2000, S. 1238 (1241 f.; der Autor ist Leiter des Referats Verwaltungsrecht und Verwaltungsverfahrensrecht im Bundesministerium des Innern).

12 Das Bundesinnenministerium hat zwei Gutachten eingeholt zu den Themen wErfordernis verwaltungsrechtlicher Kooperationsvernältnisse (Public Private Partnership)《 bei Schuppert bzw. "Verankerung verwaltungsrechtlicher Kooperationsverhältnisse (Public Private Partnership) im VwVfG « bei Ziekow. Auch diese Gutachten sind im Internet abrufbar, und zwar unter www.staat-modern.de/projekte.

13 Zu Begriff und Funktionen der Rechtsdogmatik speziell im Verwaltungsrecht vgl. Brohm, Die Dogmatik des Verwaltungsrechts vor den Gegenwartsaufgaben der Verwaltung, VVDStRL 30 (1972), S. 245 (246 f.); Schulte, Schlichtes Verwaltungshandeln, 1995, S. 9 ff.; Schmidt-Aßmann, Das allgemeine Verwaltungsrecht als Ordnungsidee, 1998, S. 4 ff.; Burgi, Funktionale Privatisierung und Verwaltungshilfe, 1999, S. 13 f, wo zwischen Systematik und Dogmatik unterschieden wird und zur Kennzeichnung dessen, was bei den soeben genannten Autoren mit $»$ Dogmatik« bezeichnet wird, nämlich der Versuch, »für größere Regelungsbereiche Vergleichbarkeiten, Zusammenhänge und Strukturen aufzuzeigen« (Schmidt-Aßmann, aaO, S. 5 in Fn. 14) der Begriff $» S y s t e m a t i k$ « verwendet wird.

14 Fleiner, Institutionen des Deutschen Verwaltungsrechts, 8. Aufl. 1928, S. 44 f;; vgl. ferner Badura, Das Verwaltungsrecht des liberalen Rechtsstaates, 1967. 
Den Gegenstand dieser Bemühungen bildet nicht der Gesamtbereich von Privatisierung und regulierter Selbstregulierung, sondern die diesbezüglichen Verfahrensfragen. Thnen gerade in diesem Zusammenhang mehr Aufmerksamkeit zu schenken, liegt aus einer steuerungswissenschaftlichen Perspektive ${ }^{15}$ besonders nahe, bezeichnen doch beide Kategorien, das »Verfahren« wie die »privatisierungsbegleitende Regulierung « (zu den Begriffen vgl. sogleich B I - III), Steuerungsinstrumente ersten Ranges, die es mit einer aufgaben- und funktionsorientierten Sichtweise zu erfassen gilt. Ausgehend von der allgemein anerkannten ${ }^{16}$ Charakterisierung des Verwaltungsverfahrens als Realvorgang und Ordnungsidee ist zu beschreiben, wie sich der Realvorgang verändert, wenn private Träger in die Gemeinwohlverwirklichung eingeschaltet werden; sodann geht es um Inhalt und Funktionen des Ordnungsmodells »Verfahren «, in dem »die vielfältigen . . . Begegnungsvorgänge zwischen Staat und Individuum oder gesellschaftlichen Gruppen ${ }^{17}$ erfasst sind und in dem nun, nach erfolgter Privatisierung bzw. Selbstregulierung, die einzelnen Verfahrensrechtsverhältnisse neu geordnet und zugeordnet werden müssen. Der Auftrag lautet daher: Funktionsbeschreibung (C u. D), Untersuchung und Ausgestaltung konkreter Verfahrensrechte und -pflichten nach Privatisierung, Benennung von Verfahrensrechtsverhältnissen, Legitimationsgrundlagen und Umsetzungsmöglichkeiten ( $E$ und F), konkret auch im Hinblick auf den

15 Charakteristikum und Stärke eines steuerungswissenschaftlichen Ansatzes, welcher das von Schmidt-Aßmann und Hoffmann-Riem forcierte Reformprojekt des Verwaltungsrechts prägt (näher geschildert bei Voßkuhle, Die Reform des Verwaltungsrechts als Projekt der Wissenschaft, DV 32 [1999], S. 545 [546 f.] m.w.N.), bestehen in der Einbeziehung der Wirksamkeitsbedingungen des Rechts und der damit verbundenen Überwindung der Perspektive einer rein hermeneutisch orientierten Normwissenschaft. Dies ist näher ausgeführt u.a. bei Schuppert, Verwaltungsrechtswissenschaft als Steuerungswissenschaft. Zur Steuerung des Verwaltungshandelns durch Verwaltungsrecht, in: Hoffmann-Riem/Schmidt-ABmann/ Schuppert (Hrsg.), Reform des Allgemeinen Verwaltungsrechts, 1993, S. 65 ff.; SchmidtAßmann, Ordnungsidee (Fn. 13), S. 18 ff., dort auch zu den Grenzen dieses Ansatzes, bei deren Respektierung die in neuerer Zeit erhobenen kritischen Einwände (Möllers, Braucht das öffentliche Recht einen neuen Methodenstreit?, VerwArch 89 [1999], S. 187 [203 ff.]; krit. hierzu sub specie des Privatverfahrensrechts bereits Burgi, DV 33 [2000], S. 196 in Fn. 64; Lepsius, Steuerungsdiskussion, Systemtheorie und Parlamentarismuskritik, 1999, S. 10 ff., $35 \mathrm{ff} ., 52 \mathrm{ff}$.) an Gewicht verlieren. Der steuerungswissenschaftliche Ansatz erzwingt konkret im vorliegenden Zusammenhang die Einbeziehung des Verfahrens als Realvorgang (vgl. C und D). Dazu, dass das Verwaltungsverfahren seinerseits ein Steuenungsinstrument ist vgl. Schuppert, Verwaltungswissenschaft, 2000, S. 772 ff.; krit. jüngst Laubinger, Der Verfahrensgedanke im Verwaltungsrecht, in: König/Merten (Hrsg.), Verfahrensrecht in Verwaltung und Verwaltungsgerichtsbarkeit, 2000, S. 47 (53 f.).

16 Ausführlich dargelegt bei Schmidt-Aßmann, Der Verfahrensgedanke in der Dogmatik des öffentlichen Rechts, in: Lerche/Schmitt Glaeser/Schmidt-Aßmann (Hrsg.), Verfahren als staats- und verwaltungsrechtliche Kategorie, 1984, S. 1 ( 6 ff.); ferner Hufen, Fehler im Verwaltungsverfahren, 3. Aufl. 1998, Rn. 46 f;; Schoch, Der Verfahrensgedanke im Allgemeinen Verwaltungsrecht, DV 25 (1992), S. 21 ff.

17 Schmidt-Aßmann, in: Verfahrensgedanke (Fn. 16), S. 7. 
bereits erwähnten rechtspolitischen Prozess der Schaffung eines Rechtsrahmens.

Freilich stellt sich die Frage nach der »Funktion des Verfahrens « in »privatisierten Bereichen« nicht in allen Bereichen mit gleicher Intensität bzw. mit gleichem Inhalt. Knüpft man an die allgemein anerkannte Begriffsbestimmung des Verwaltungsverfahrens an und lässt die beiden Begriffsmerkmale weg, die in Privatisierungszusammenhängen ganz oder teilweise unzutreffend sind (dass ein Träger öffentlicher Verwaltung das Verfahren alleine führt und die Entscheidungen alleine trifft), dann geht es jedenfalls um "planvoll geordnete Vorgänge der Informationsgewinnung und -verarbeitung . . . die der Hervorbringung von Entscheidungen dienen ${ }^{18}{ }^{18}$. Daraus folgt, dass diejenigen Erscheinungsformen der Privatisierung bzw. regulierten Selbstregulierung, in deren Gefolge es um entscheidungsbezogene Informationsgewinnung und -verarbeitung allenfalls am Rande geht, vielmehr technische Durchführung ${ }^{19}$ (wie bei den Betreibermodellen im Infrastrukturbereich) oder definitionsgemäße Dienstleistungserbringung (wie beim sog. Dualen System im Abfallrecht oder bei den Universaldienstleistungspflichten [vgl. z.B. $\S \S 17$ ff. TKG]) gefordert sind, wegen fehlendem Verfahrensbezug außen vor bleiben können. Verfahrensfragen stellen sich insoweit lediglich im Verhältnis zwischen dem Staat und den jeweiligen privaten Trägern, und zwar im Zusammenhang mit deren Veranlassung sowie im weiteren Verlaufe der Kooperation (z.B.: Auskunfts- und Informationspflichten); sie können indes anhand der allgemeinen Regeln des Vergaberechts bzw. im Rahmen der Kooperationsbeziehung bewältigt werden. Im Mittelpunkt der folgenden Ausführungen müssen diejenigen »privatisierten Bereiche« stehen, in deren Mittelpunkt Prozesse der entscheidungsbezogenen Informationsgewinnung und -verarbeitung ablaufen.

18 Nach Hill, Das fehlerhafte Verfahren und seine Folgen im Verwaltungsrecht, 1986, S. 193 ff.; Schmidt-Aßmann, Verwaltungsverfahren, in: Isensee/Kirchhof (Hrsg.), HdbStR JIl, 1988 , § 70 Rn. 1; Wolff/Bachof/Stober, Verwaltungsrecht II, 6. Aufl. 2000, 58 Rn. 1. Der Fokus der folgenden Betrachtungen liegt mithin auf den Entscheidungsverfahren, die bislang ganz im Vordergrund des verwaltungsrechtlichen Interesses und der verwaltungsrechtlichen Dogmatik gestanden haben. Damit wird nicht geleugnet, dass es andere Verfahrenstypen gibt, im Hinblick auf die ein rechtsdogmatischer Nachholbedarf bestehen mag (vgl. Schmidt-Aßmann, Ordnungsidee [Fn. 13], S. 288 f.). Angesichts des Anfangsstadiums, in dem sich das Verfahrensrecht nach Privatisierung gegenwärtig befindet, erscheint aber empfehlenswert, nach funktionalen Äquivalenten erst einmal (und nur) dort zu suchen, wo Begriff und Funktion des Verwaltungsverfahrens im Normalfall des staatlich verantworteten Verfahrens gefestigt sind, mithin im Bereich der Entscheidungsverfahren.

19 In Anknüpfung an die bereits in meiner Arbeit wFunktionale Privatisienung und Verwaltungshilfe «, 1999, S. 100 ff., entfaltete Unterscheidung von Durchführungsprivatisierung und Vorbereitungsprivatisierung, jedoch darüber hinaus gehend (vgl. sogleich B I - III). 


\section{B. Grobstrukturierung der "privatisierten Bereiche»}

Die Termini »Privatisierung $\aleph^{20}, »$ Verantwortungsteilung $\aleph^{21}$, $»$ Regulierte Selbstregulierung $\aleph^{22}$, »Public Private Partnerships $\aleph^{23}-$ um nur die Begriffe mit der größten Breitenwirkung zu nennen - kehren in der bisherigen Diskussion in immer neuen und sich vielfach überlappenden Zusammenhängen wieder. Dies mag in der Phase »der Ideenformulierung, des Experimentierens und Ausprobierens $\aleph^{24}$ akzeptabel und hilfreich gewesen sein, wird aber nun, beim Übergang zur dogmatischen Phase, zum klärungsbedürftigen Problem.

Emigkeit dürfte $\mathrm{m}$. E. über den zu erfassenden Gesamtbereich zu erzielen sein, der im "Grenzbereich zwischen staatlichen und privaten Aktivitäten" liegt und gekennzeichnet ist durch staatlich induzierte Veränderungen in der Arbeitsteilung bei der Erfüllung von Aufgaben im öffentlichen Interesse ${ }^{25}$, anders formuliert, durch ein neues Mischungsverhältnis zwischen staatlicher und gesellschaftlicher Handlungsrationalität. Dahinter stehen (mittlerweile fast schon Gemeingut gewordene) politische Zielsetzungen (v.a. Staatsentlastung, Mobilisierung gesellschaftlicher Potentiale zur Verbesserung der Aufgabenerfüllung, aber auch im eigenen Freiheitsinteresse der gesellschaftlichen Träger), die zu respektieren sind und im Verlaufe der dogmatischen Folgenbewältigung nicht konterkariert werden dürfen. Schon aus diesem Grund wird es kaum möglich sein, verfahrensrechtliche Anforderungen im Verhältnis eins zu eins zu übertragen, wie überhaupt perspektivische Verengungen (sei es in Richtung »Regulierung«, sei es in Richtung der vielbeschworenen »Kräfte des Marktes«) zu vermeiden sind.

20 Etwa verwendet bei Ramsauer, VwVfG, 7. Aufl. 2000, Einführung Rn. 92 ff.; Stelkens/ Schmitz, in: Stelkens/Bonk/Sachs (Hrsg.), VwVfG, 6. Aufl. 2001, § 1 Rn. 101 ff.; vgl. ferner die neueren Habilitationsarbeiten von Burgi (Fn. 13), Gramm, Privatisierung und notwendige Staatsaufgaben, 2001, und Kämmerer, Privatisierung, 2001.

21 Maßgeblich geprägt und weiterentwickelt durch die Beiträge von Trute, in: Veranwortungsteilung (Fn. 5), S. 13 ff., und Voßkuhle, aaO, S. 47 ff.; vgl. ferner Schmidt, Die Reform von Verwaltung und Verwaltungsrecht, VerwArch 91 (2000), S. 149 (157 f.); krit. Röhl, DV 1999, Beiheft 2, S. 33 ff; Kämmerer (Fn. 20), S. 433 ff.

22 Damit haben sich im Anschluss an die Vorträge von Schmidt-Preuß und Di Fabio auf der Staatsrechtslehrertagung 1996 (VVDStRL 56 [1997], S. 160 ff., 235 ff.) u.a. befasst Hoffmann-Riem, in: Öffentliches Recht und Privatrecht (Fn. 2), S. 300 ff. m.w.N. aus früheren Arbeiten. Entscheidend verbreitert und vertieft worden sind die sich hiermit verbindenden Vorstellungen durch die zu B III verarbeiteten Beiträge auf dem gleichnamigen Symposium aus Anlass des 60. Geburtstages von Hoffmann-Riem (Fn. 6).

23 Vgl. zu diesem schillernden Begriff, der Aufnahme in den Titel der beiden erwähnten Gutachten für das Bundesinnenministerium gefunden hat (Fn. 12) nur Burgi (Fn. 13), S. 98 f., sowie bereits (mit krit. Tendenz) Tettinger, Die rechtliche Ausgestaltung von Public Private Partnership, DÖV 1996, S. 764, sowie die Beiträge in Budäus/Eichhorn (Hrsg.), Public Private Partnership, 1997.

24 Formulierung nach Voßkuhle, VerwArch 92 (2001), S. 215.

25 Formulierung nach Schmidt-ABmann, DV 2001, Beiheft 4, S. 254; vgl. auch Ziekow (Fn. 12), II 1 . 
Der soeben skizzierte Gesamtbereich wird m. E. am treffendsten mit dem heuristischen Begriff der "Verantwortungsteilung u umschrieben, durch den der Übergang von der Erfüllungsverantwortung bzw. von der vollkommenen staatlichen Abstinenz zur sog. Gewährleistungsverantwortung des Staates zum Ausdruck gelangt ${ }^{26}$. Die hiervon betroffenen Bereiche sind »privatisierte Bereiche « im Sinne der hiesigen Themenstellung, wenn man »Privatisierung « auf die Aufgabenverantwortung und nicht auf Organisation oder Vermögen bezieht; Vorgänge der bloßen Organisationsprivatisierung einschließlich die Beleihung bleiben daher ebenso ausgeblendet wie die Veräußerung von Vermögensanteilen (wie sie etwa in den Kontexten der Art. 87e u. 87f GG stattfindet) ${ }^{27}$. Wie sogleich gezeigt wird, lassen sich in Gestalt der (regulierten) Verwaltungshilfe und der regulierten Selbstregulierung zwei große Teilbereiche unterscheiden und verschiedene weitere Bereiche thematisch ausgrenzen. Der seinerseits heuristische Begriff »Public Private Partnership« bezieht sich auf die Kooperation als wichtigstem (aber nicht einzigstem) ${ }^{28}$ »Verwirklichungsmodus« von Verantwortungsteilungen, mit einer Verengung auf den Bereich rechtlich fixierter, administrativ herbeigeführter Kooperationen ${ }^{29}$. In Begleitung und im Anschluss an eine Verantwortungsteilung (respektive eine

26 Insoweit kann man von einer »Grundkategorie« sprechen (in Anlehnung an Pitschas, Verwaltungsverantwortung und Verwaltungsverfahren, 1990, S. 9, 240 ff.; vgl. ferner Vo $\beta$ kuhle, in: Verantwortungsteilung, S. 52 ff.; Ziekow [Fn. 12], I 2, S. 4 f.). Dabei wird angeknüpft an das seinerseits heuristische Konzept der Bildung von Verantwortungsstufen (vgl. nur $S c h m i d t-A \beta m a n n$, in: Reform [Fn. 15], S. 43 f. u. S. 60 f.); mit dem Begriff der $» G e-$ währleistungsverantwortung « wird bezeichnet, dass der Staat wmit Hilfe der rechtlich bereit gestellten Strukturen angemessene gesellschaftliche Problemlösungen« zu erreichen ermöglicht (Hoffmann-Riem, Tendenzen in der Verwaltungsrechtsentwicklung, DÖV 1997, S. 433; vgl. ferner Schuppert, DV 2001, Beiheft 4, S. 201 ( 218 ff. m.w.N.). Hiermit verbinden sich weder auf der Ebene des Verfassungsrechts noch auf der des einfachen Rechts bestimmte Rechtsfolgen, insbesondere lassen sich allein mit der Zuordnung bestimmter privater Handlungsbeiträge zu einer bestimmten Verantwortungsstufe nicht Pflichtenstellungen über das allgemein verbindliche $\mathrm{MaB}$ hinaus begründen. Auch an der Unterscheidung von Staat und Gesellschaft im freiheitlichen Verfassungsstaat (vgl. nur Rupp, Die Unterscheidung von Staat und Gesellschaft, in: Isensee/Kirchhof [Hrsg.], HdbStR I, 1987, § 28 Rn. 25 ff.) ändert sich nichts. So bedarf auch die Begründung von Verfahrenspflichten erheblicher dogmatischer Anstrengung (vgl. E I) jenseits der Ebene der Begriffsbildung.

$27 \mathrm{Zu}$ diesen Unterscheidungen und zu der im Zuge der Bahn- und Postprivatisierung zusätzlich in Gang gesetzten $» D e r e g u l i e r u n g "$ (im Sinne eines Abbaus von Monopolstellungen) vgl. nur Burgi (Fn. 13), S. 2 ff., 9 ff.

28 Neben der zwangsweisen Veranlassung zur Erbringung von Verwaltungshelferdiensten (etwa in Gestalt des Lohnsteuereinzugs durch die Arbeitgeber (vgl. hierzu Burgi [Fn. 13], S. $253 \mathrm{ff}$.) und der $z w a n g s w e i s e n$ "Inpflichtnahme« für eine selbstregulierende Tätigkeit (etwa in Gestalt der Eigenüberwachung im Umweltrecht [vgl. sogleich B III I b]; zu beiden eingehend, mit zahlreichen Belegen aus der teilweise Jahrzehnte zurückreichenden Literatur Burgi [Fn. 13], S. 90 ff.) gibt es Erscheinungsformen von Verantwortungsteilungen, bei denen zwar die private Mitwirkung nicht angeordnet wird, bei denen es aber auch nicht zu einem rechtlich fixierten Kooperationsakt kommt, wie etwa im Falle des Öko-Audit (vgl. dazu sogleich B III 1 b).

Im Anschluss an Ziekow (Fn. 12), I 2, S. 4 f. 
aufgabenbezogene Privatisierung) erfolgen Maßnahmen der »Regulierung « durch den Staat, welche die »Gewährleistungsverantwortung « (respektive die »Privatisierungsfolgenverantwortung «) normativ und/oder administrativ umsetzen $^{30}$.

\section{II. (Regulierte) Verwaltungshilfe (nach funktionaler Privatisierung)}

Die Verwaltungshilfe - hierbei handelt es sich um eine bereits dogmatische Kategorie - entsteht durch eine sog. funktionale Privatisierung, bei der ein Teil der staatlichen Verantwortung für die Erfüllung einer bestimmten Aufgabe als Staatsaufgabe abgespalten wird. Der Verwaltungshelfer erbringt für den Staat und unter dessen Leitung auf der Basis eines "Veranlassungsaktes « einen Teilbeitrag, welcher funktional auf die Staatsaufgabe bezogen ist ${ }^{31}$. Jener Teilbeitrag kann durchführenden Charakters sein, sich also auf eine bereits getroffene staatliche Entscheidung beziehen; er besteht dann typischerweise in der Erbringung von Dienstleistungen technischen oder finanziellen Charakters, was mit dem Hinweis auf die bekannten Figuren des Betreibers bzw. Betriebsführers in der Infrastrukturverwaltung illustriert werden kann. Unter Verfahrensaspekten wichtiger (vgl. A II) sind die Vorbereitungshelfer, die planerische ${ }^{32}$, sachverständig-beratende ${ }^{33}$ oder auch Unterstützungsbeiträge innerhalb eines Verwaltungsverfahrens (als sog. Verfahrensmittler) ${ }^{34}$ erbringen und dadurch

30 Wenngleich sich der Regulierungsbegriff nicht auf diesen Bereich beschränkt, sondern alle hoheitlich wahrgenommenen Tätigkeiten des Staates smit einem spezifischen, über den Einzelfall hinausgreifenden Ordnungszweck « umfasst (Schmidt-Aßmann, DV 2001, Heft 4, S. 255), befindet sich doch in den vorliegenden Zusammenhängen seine (gegenwärtig) wichtigste Heimat (hierzu vgl. im Anschluss an Lerche, in: Maunz/Dürig, GG, Stand März 2001, Art. 87f Rn. 34 f., Ruffert, Regulierung im System des Verwaltungsrechts, AöR 124 [1999], S. 237 [246]; Schneider, Liberalisierung der Stromwirtschaft durch regulative Marktorganisation, 1999, S. 41 ff. Zum Begriff »Privatisierungsfolgenverantwortung « vgl. Bauer, Privatisierung von Verwaltungsaufgaben, VVDStRL 54 (1995), S. 243 ff. (279); Burgi, Kommunales Privatisierungsfolgenrecht, NVwZ 2001, 601 ff., sowie mit teilweise krit. Tendenz in begrifflicher Hinsicht Kämmerer (Fn. 20), S. 479 ff., 489 ff.

31 Dieser funktionale Bezug ist entscheidend, nicht, ob der erbrachte Beitrag selbständig oder unselbständig ist bzw. ob die Person des Verwaltungshelfers selbständig oder unselbständig ist (ausführlich dargelegt bei Burgi [Fn. 13], S. 145 ff.; vgl. ferner bereits Schoch, Privatisierung von Verwaltungsaufgaben, DVB1. 1994, S. 962 [963], sowie jüngst Schmidt-Aßmann, DV 2001, Beiheft 4, S. 261).

32 Beispiele: Private Architektur- und Ingenieurbüros auf der Grundlage des $\S 11$ Abs. 1 Ziffer 1 BauGB, Private, die Standortalternativen im Zuge der Planung von Infrastrukturanlagen sammeln und aufbereiten (vgl. nur Burgi, DV 33 [2000], S. 187 f. m.w.N.).

33 Beispiele können fast allen wichtigen Aufgabenfeldern entnommen werden, mit einem gewissen Schwerpunkt in Bereichen mit naturwissenschaftlich-technischem Hintergrund; vgl. hierzu bereits Brohm, Sachverständige Beratung des Staates, in: Isensee/Kirchhof (Hrsg.), HdbStR II, 1987, §36; Burgi (Fn. 13), S. 130 ff.; Seidel (Fn. 10), S. 215 ff.

34 Sofern nicht Konstellationen der Beleihung bzw. der regulierten Selbstregulienung vorliegen. Anwendungsbeispiele sind vor allem im Vollzug des $\$ 2$ Abs. 2 Satz 3 Ziffer 5 der 9. BImSchVO (vgl. hierzu Ludwig, Privatisierung staatlicher Aufgaben im Umweltschutz, 1998, S. 198 ff.) bzw. des § 4b BauGB anzutreffen (vgl. hierzu vorerst nur Reidt, § 4b 
staatliche Entscheidungen determinieren können. Bei ihnen handelt es sich um Dritte, die namentlich im Bau- und Fachplanungsrecht sowie im Umweltrecht Material erheben, sammeln und auswerten oder auch hieraus Schlüsse ziehen, ihre Erkenntnisse aber verabredungsgemäß den zuständigen staatlichen Stellen buchstäblich überantworten, zur Erledigung der jeweiligen Staatsaufgabe innerhalb des hierfür vorgesehenen Verfahrensrahmens.

\section{Regulierte Selbstregulierung}

\section{Begriff und Spektrum}

Hierbei handelt es sich um einen gegenwärtig noch heuristischen Begriff, der auf seinem noch weiten Weg in die verwaltungsrechtliche Dogmatik ${ }^{35}$ vorliegend nur ein kleines Stück weit begleitet werden kann, nämlich in verfahrensbezogener Perspektive. Unzulänglichkeiten, die sich aus der gegenwärtig nicht möglichen Einbettung in ein dogmatisches Gesamtkonzept (vergleichbar dem der Verwaltungshilfe) ergeben können, müssen in Kauf genommen werden. Worum geht es? Um die Mobilisierung gesellschaftlicher Kräfte, welche sich zwar in ihrer eigenen Handlungsrationalität bewegen und durch spezifische Dienstleistungen eine eigene kollektive Ordnung hervorbringen, deren Tätigwerden also nicht funktional auf eine Staatsaufgabe bezogen, aber auch nicht »schlicht gesellschaftlich« und lediglich der allgemeinen Aufsicht unterworfen ist. Vielmehr vollzieht es sich innerhalb eines staatlich gesetzten Rahmens, innerhalb eines gemeinsamen Ordnungssystems ${ }^{36}$, dessen Ausge-

BauGB - Die Einschaltung Dritter in die Bauleitplanung, NVwZ 1998, 592) und, ohne explizite gesetzliche Grundlage, in zahlreichen weiteren Bereichen mit komplexen Verfahren (vgl. näher Burgi, DV 33 [2000], S. 186 f; Seidel [Fn. 10], S. 247 ff.). Die Möglichkeiten und Grenzen innerhalb weiterer Bereiche sind illustriert in den Beiträgen von Peine, Ronellenfitsch, Schwarz, Köchling und Hofmann-Hoeppel, in: Verfahrensprivatisierung (Fn. 4), S. 95 ff., 113 ff., 188 ff., 201 ff., 216 ff. In \& 98 Abs. 2 des Entwurfs eines Umweltgesetzbuchs ist vorgesehen, dass die Genehmigungsbehörde »die Durchführung einzelner Abschnitte des Verfahrens ... e einem Verfahrensmittler . . . übertragen« kann (vgl. die Begründung in: Bundesministerium für Umwelt, Naturschutz und Reaktorsicherheit [Hrsg.], Entwurf der Unabhängigen Sachverständigenkommission zum Umweltgesetzbuch, 1998, S. 640 ff.). Hierher können ferner auch Private gerechnet werden, die mit der Vornahme von Untersuchungen, Erhebungen oder Auswertungen betraut sind, etwa auf der Grundlage des $\S 40$ Abs. $2 \mathrm{KrW}-/$ AbfG (näher hierzu Ludwig [Fn. 34], S. 165 ff.; Burgi [Fn. 13], S. 140 f.).

35 Den gegenwärtigen Erkenntnis- und Entwicklungsstand hat jüngst Schmidt-Aßmann, DV 2001, Beiheft 4, S. 254 ff., 271, fixiert, anknüpfend an Hoffmann-Riem, in: Offentliches Recht und Privatrecht (Fn. 2), S. 300 ff.; Trute, Die Verwaltung und das Verwaltungsrecht zwischen gesellschaftlicher Selbstregulierung und staatlicher Steuerung, DVB1. 1996, S. 950 ff.; Schuppert, DV 31 (1998), S. 415 ff.; vgl. ferner Burgi (Fn. 13), S, 87 ff., sowie Voßkuhle, DV 2001, Beiheft 4, S. 197 ff. Dogmatische Ansätze finden sich auch bei Faber, Gesellschaftliche Selbstregulierungssysteme im Umweltrecht - unter besonderer Berücksichtigung der Selbstverpflichtungen, 2001, S. $8 \mathrm{ff}$.

36 Wie Schmidt-Aßmann, DV 2001, Beiheft 4, S. 255, hervorgehoben hat, ist das zentrale Charakteristikum »der Verbund« zwischen Selbstregulierung und Regulierung. 
staltung ebenso vielgestaltig ist, wie es die Motive und die Referenzgebiete sind. Das Instrumentarium der staatlichen Regulierung in diesem Bereich erschöpft sich wiederum nicht in »Initiierung, Begleitung und Absicherung ${ }^{37}$, mithin in Kooperation, sondern umschließt auch die zwangsweise »Inpflichtnahme« für das Gemeinwohl, etwa bei der Pflicht zur Eigenüberwachung im Umweltrecht ${ }^{38}$.

Zusammenfassend lässt sich festhalten, dass ein Fall der »regulierten Selbstregulierung « dann vorliegt, wenn gesellschaftliches Handeln durch Einbeziehung in einen gemeinsamen Ordnungsrabmen mit dem Staat für das Gemeinwohl nutzbar gemacht wird. Einige der wichtigen Erscheinungsformen regulierter Selbstregulierung wurzeln bereits in einem europäisierten Verwaltungskontext (vgl. sogleich im Text) und es ist damit zu rechnen, dass das Konzept als solches europäischen Rückenwind erhält, wenn die Vorstellungen der EU-Kommission in ihrem im Mai 2001 vorgestellten Weißbuch $» E u-$ ropäisches Regieren « verwirklicht werden. ${ }^{39}$ Danach sollen im Rahmen einer stärkeren »Einbindung der Zivilgesellschaft« (III, 3.1., S. 19 ff.) »Partnerschaftsregelungen ... mit Organisationen der Zivilgesellschaft « entwickelt werden (S. 22), verstärkt "Expertenwissen« eingeholt und genutzt werden (III, 3.2., S. 25 f.) und die "Koregulierung « verstärkt werden, allerdings unter der Voraussetzung, dass die beteiligten Organisationen in der Lage sind, »sich in offenen Verfahren an vereinbarte Regeln zu halten« (III, 3.2., S. 27 f.).

Aufgrund der Vielzahl und Vielfalt der Erscheinungsformen ist eine auch nur annähernd vollständige Erfassung des Spektrums - zumal im gegebenen Rahmen nicht möglich. Für die Zwecke der nachfolgenden verfahrensorientierten Betrachtung seien einige wichtige Beispiele aus verschiedenen »Referenzgebieten « genannt: ${ }^{40}$

37 Voßkuhle, VerwArch 92 (2001), S. 214.

38 Vgl. zu diesem und zu weiteren Beispielen sogleich im Text. Die klassischen Anwendungsfälle der früheren Diskussionen um đie Verfassungsmäßigkeit von »lnpflichtnahme« bzw. »Indienstnahme«, nämlich die Pflicht zur Erdölbevorratung bzw. zur Übernahme des Räumund Streudienstes an öffentlichen Wegen durch die Anlieger (näher dargestellt mit zahlreichen Nachweisen bei Burgi [Fn. 13], S. 90 ff.) lassen sich ebenfalls dem Spektrum der regulierten Selbstregulierung zuordnen.

39 Vom 25.7.2001, KOM (2001), 428; eine erste Einschätzung auf der Basis der vorbereitenden Überlegungen bei Burgi, Vertikale Kompetenzabgrenzung in der EU und materiellrechtliche Kompetenzausübungsschranken nationaler Daseinsvorsorge, in: Henneke (Hrsg.), Verantwortungsteilung zwischen Kommunen, Ländern, Bund und Europäischer Union, 2001, S. $90 \mathrm{ff}$.

40 Weitere Referenzbereiche werden thematisiert durch Holznagel, Regulierte Selbstregulierung im Medienrecht, S. 81 ff., Schulz, Regulierte Selbstregulierung im Telekommunikationsrecht, S. $101 \mathrm{ff}$., Eifert, Regulierte Selbstregulierung und die lernende Verwaltung, S. $137 \mathrm{ff}$., Schuler/Harms, Regulierte Selbstregulierung im Polizei- und Versammlungsrecht, S. 159 ff., Schneider, Regulierung staatsinterner Selbstregulierung am Beispiel des Haushaltswesens, S. 177 ff., jeweils in DV 2001, Beiheft 4, sowie bei Schmidt-Aßmann, aaO, S. $256 \mathrm{f}$. 
a) Das klassische Referenzgebiet Baurecht bietet ein erstes Beispiel in Gestalt der reformierten Landesbauordnungen, die unter bestimmten Voraussetzungen auf das Baugenehmigungserfordernis verzichten und die Verantwortung für die Einhaltung des unverändert beachtlichen materiellen Baurechts vollständig oder teilweise in die Hände des Bauherren und der durch diesen zu bestellenden Sachverständigen legen (vgl. z.B. $§ 67$ BauO NRW) ${ }^{41}$; eine weitere Erscheinungsform aus dem Baurecht bildet der sog. Vorhaben- und Erschließungsplan nach $\S 12$ BauGB, der die Vorstellungen des privaten Investors und Vorhabenträgers bündelt, welche sich die Gemeinde sodann mit einem »vorhabenbezogenen Bebauungsplan« zu eigen machen kann. Voraussetzung hierfür ist der Abschluss eines Durchführungsvertrages, mit dem sich der private Vorhabenträger zur Verwirklichung der entsprechenden Festsetzungen verpflichtet ${ }^{42}$.

b) Aus dem Umweltrecht ${ }^{43}$ seien die mit »Eigenüberwachung « bezeichneten Verantwortungsstrukturen genannt, deren Elemente die schlichten Dokumentations- und Kontrollpflichten der Anlagenbetreiber, die Schaffung einer Umweltbeauftragtenorganisation (vgl. z.B. $\S \S 53 \mathrm{f}$. BImSchG) und das komplexe, europäisch inspirierte System des Öko-Audits bilden ${ }^{44}$. Ging es bei diesem zunächst um den freiwilligen Aufbau eines Verpflichtungs-, Management- und Prüfungssystem auf Unternehmensebene mit abschließender Registereintragung (vgl. §33 Umweltauditgesetz), so sind zwischenzeitlich Ansätze entwickelt worden, um über vereinbarte Substitutionskataloge bzw. Öffnungsklauseln in Verwaltungsvorschriften oder Gesetzen (ein VO-Entwurf des Bundesumweltministerium existiert bereits) Vollzugserleichterungen ${ }^{45}$ bei der staatlichen Anlagenüberwachung zu bewirken.

41 Ein annähernd aktueller Überblick über das Regelungsspektrum in den einzelnen Bundesländern findet sich bei Hoppe/Grotefels, Öffentliches Baurecht, $\S 15$.Zur Einordnung in den Kontext der regulierten Selbstregulienung vgl. Schmidt-Preuß, VVDStRL 56 (1997), S. $194 \mathrm{ff}$., sowie, mit Fokus auf die eingeschalteten Sachverständigen Seidel (Fn. 10), S. $262 \mathrm{ff}$.

42 Vgl. hierzu nur Schneider, Kooperative Verwaltungsverfahren, VerwArch 87 (1996), S. 38 (41 ff., 56 ff.); Brohm, Offentliches Baurecht, 3. Aufl. 2002, 87 Rn. 23 ff.; Turiaux, Der vorhabenbezogene Bebauungsplan gem. $\S 12$ BauGB: Beschleunigungspotenzial, Durchführungsverpflichtung und praktische Probleme, NJW 1999, S. 391; Faber (Fn. 35), S. $128 \mathrm{ff}$.

43 Instruktive Gesamtdarstellungen bei Laskowski, in: Verantwortungsteilung (Fn. 5), S. 93 ff., und Brandt, DV 2001, Beiheft 4, S. 123 ff. Unter dem Aspekt der Etablierung von Organisationsanforderungen gegenüber privaten Unternehmen jüngst Spindler, Unternehmensorganisationspflichten, 2001, S. $328 \mathrm{ff}$.

44 Aus der überbordenden Literatur vgl. sub specie des allgemeinen Privatisierungskontextes nur Schneider, Öko-Audit als Scharnier einer ganzheitlichen Regulierungsstrategie, DV 28 (1995), S. 361; Schmidt-Aßmann, DV 2001, Beiheft 4, S. 256; Schickert, Der Umweltgutachter der EG-Umwelt-Audit-Verordnung, 2001; Kämmerer, Die Umsetzung des UmweltAudit-Rechts, 2001.

45 Zu ersten Ansätzen der Einbettung des Öko-Audit in ein System der Substitution staatlicher Ordnungspflichten in Bayem vgl. Böhm-Amtmann, Umweltpaket Bayern - Miteinander die 
c) Im ebenfalls europäisch determinierten Produktsicherheitsrecht agieren auf der administrativen Ebene neben den Herstellern und privaten Sachverständigen die sog. Benannten Stellen, die nach Akkreditierung durch den Staat aufgrund eines Vertrages mit dem Produkthersteller die Konformität von dessen Produkt mit den Anforderungen der jeweils einschlägigen EGRichtlinie beurteilen und ohne deren Zertifizierung das Produkt nicht in den Verkehr gebracht werden kann (vgl. z.B. $\$ 9$ GSG) ${ }^{46}$. Mit Zertifizierung ist das Produkt EG-weit verkehrsfähig und würden weitere Zulassungsverfahren oder systematische Kontrollen durch mitgliedstaatliche Behörden grundsätzlich unzulässig.

d) Aus dem Wirtschaftsverwaltungsrecht bildet die durch $\S 25 \mathrm{a}$ Abs. 1 KWG eingeführte Pflicht zum Aufbau eines Risikomanagement-, -controlling und -informationssystems ein aktuelles und wirtschaftlich hochbedeutsames Beispiel im Grenzbereich von staatlicher Bankenaufsicht und privater Bankorganisation. Hierdurch werden Organisation und Verfahren bei der Steuerung und Kontrolle von Risiken des Bankgeschäfts auf das Gemeinwohl, konkret auf die mit der Bankenaufsicht gemäß $\S 6$ Abs. 2 KWG verfolgten Ziele ausgerichtet. Der Aufbau eines Risk Management-Systems ist als Voraussetzung für die Erteilung der Erlaubnis für das Betreiben von Bankgeschäften ausgestaltet ( $\$ 33$ Abs. 1 Satz $1 \mathrm{Nr} .7 \mathrm{KWG}$ ), so dass, im Unterscheid etwa zum Öko-Audit bisheriger Prägung, von vornherein eine Verzahnung mit der behördlichen Vollzugstätigkeit besteht ${ }^{47}$.

Umwelt schützen, ZUR 1997, S. 178; Schuppert, Verwaltungswissenschaft (Fn. 15), S. 416 f.; Bender/Sparwasser/Engel, Umweltrecht, 4. Aufl. 2000, Kapitel 3 Rn. 45. Im Gefolge der im Februar 2000 in Kraft getretenen Novelle der Öko-Audit-Verordnung (EMASVerordnung) der EG (vom 19.3.2001; AB1. EG L 114, S. 1; vgl. hierzu Langerfeld, Die novellierte EG-Öko-Audit-Verordnung: Evolution oder Revolution?, UPR 2001, S. 220; Knopp, EMAS II - Uberleben durch »Deregulierung« und »Substitution«?, NVwZ 2001, S. 1098) hat das Bundeskabinett in seiner Sitzung vom 19.9.2001 den Entwurf einer sog. EMAS-Privilegierungs-Verordnung (EMASPrivilegV) auf der Basis des $\$ 58 \mathrm{e}$ BImSchG verabschiedet (zwischenzeitlich in Kraft getreten, EMASPrivilegV vom 24. Juni 2002, BGBl. I, S. 2247) (www.bmu.de) mit Erleichterungen bei den Berichts-, Anzeige- und Messungspflichten im Bereich der Überwachung; im Hinblick auf begünstigende Entscheidungen (Genehmigungen) kann u.U. auf die Anfordenung umfangreicher Antragsunterlagen verzichtet werden, wenn geprüfte Unterlagen aus dem Öko-Audit vorgelegt werden, die dem erforderlichen Inhalt der Anträge entsprechen (krit. hierzu bereits Knopp, NVwZ 2001, S. 1098).

46 Die Einzelheiten sind systematisch aufbereitet und als »Elemente einer Europäischen Verwaltungsstruktur« identifiziert in der grundlegenden Arbeit von Röhl, Akkreditierung und Zertifizierung im Produktsicherheitsrecht, 2000, S. 53 ff., 79 ff.; vgl. ferner Seidel (Fn. 10), S. 266 f.; Spindler (Fn. 43), S. 159 ff.

47 Vgl. hierzu Spindler (Fn. 43), S. $196 \mathrm{ff}$. (im Weiteren werden vergleichbare Bestimmungen im wirtschaftsverwaltungsrechtlichen Umfeld thematisiert); Burgi, Risk Management und Bankenaufsicht im System des Wirtschaftsverwaltungsrechts, in: Lange/Wall (Hrsg.), Risikomanagement nach dem KonTraG, 2001, S. 307 ff. Auch dort sind Kontrollerleichterungen zur Honorierung hjerbei erbrachter überobligationsmäßiger Anstrengungen denkbar (Burgi, aaO, S. 319 f., mit ersten Nachweisen). 


\section{Unterscheidungskriterium "Entscheidungsbezug»}

Anknüpfend an die eingangs angestellten Überlegungen zur Verfahrensrelevanz und zur Kennzeichnung des Verfahrens als Prozess der Informationsgewinnung und -verarbeitung mit Entscheidungsbezug (A II) soll innerhalb der Bereiche der regulierten Selbstregulierung danach unterschieden werden, ob ein Bezug zu staatlichen Entscheidungen besteht, oder ob ein solcher Bezug fehlt, dafür aber innerhalb des selbstregulierenden Sektors Prozesse der Informationsgewinnung bzw. -verarbeitung mit Entscheidungsbezug ablaufen. Dies trägt zunächst der Erkenntnis Rechnung, dass die zahlreichen und vielgestaltigen Phänomene der regulierten Selbstregulierung im Stadium der Bestimmung konkreter Rechtsfolgen nicht mehr über einen Leisten geschlagen werden können. Sodann wird die zu D unternommene Funktionsanalyse vorbereitet und die für eine Äquivalenzprüfung (Verfahrensrecht vor und nach Privatisierung) notwendige strukturelle Transparenz hergestellt. Ordnet man die soeben beispielhaft genannten Erscheinungsformen zu, so gehört das ÖkoAudit in seiner bisherigen Prägung, d. h. ohne Verzahnung mit dem Umweltordnungsrecht, zur zweiten Gruppe, indem ein komplexer Vorgang der Informationsgewinnung und -verarbeitung in die Entscheidung des privaten Umweltgutachters über die Gültigkeit der abgegebenen Umwelterklärung mündet; entsprechendes gilt im Hinblick auf das Tätigwerden anerkannter ${ }^{48}$ Prüf- und Bestätigungsstellen nach dem am 22.5.2001 in Kraft getretenen neuen Signaturgesetz ${ }^{49}$.

Die übrigen Beispielsfälle befinden sich auf der anderen Seite des Spektrums, namentlich die Zertifizierung im Produktsicherheitsrecht und das Risk-Management, aber auch das Öko-Audit künftiger Prägung, weil jeweils zwar nicht eine unmittelbare Vorbereitungstätigkeit erbracht wird, aber doch ein Bezug zu staatlicher Entscheidungsverantwortung besteht, entweder dahingehend,

48 Die Prüf- und Bestätigungsstellen sind vor allem für die Bestätigung zuständig, dass die technischen Komponenten von wProdukten für qualifizierte elektronische Signaturen« den Anforderungen des $\S 17$ Abs. 1 und 3 Ziffer 1 SignaturG entsprechen (vgl. $\S 17$ Abs. 4 Satz 1 SignaturG). Die Voraussetzungen für ihre Anerkennung seitens der »zuständigen Behörden « sind in § 18 SignaturG geregelt und entsprechen in etwa den Anforderungen an die Zulassung als Umweltgutachter nach den $\S \S 4$ u. 9 Umweltauditgesetz. Die Prüf- und Bestätigungsstellen sind gewissermaßen Implementationsinstanzen (vgl. E II vor 1) zweiter Stufe, indem sie wiederum das Verhalten der ihrerseits sakkreditierten Zertifizierungsdiensteanbieter« (vgl. \& 4 ff. SignaturG) prüfen und bestätigen. Sowohl die Entscheidungen der Zertifizierungsdiensteanbieter als auch die Entscheidungen der Prüf- und Bestätigungsstellen weisen keinen Bezug zu späteren verwaltungsbehördlichen Entscheidungen auf. Vielmehr erzeugen sie Rechtswirkungen dadurch, dass der Gesetzgeber in verschiedenen Formvorschriften (vgl. etwa \& 126a BGB) die Verwendung »elektronischer Signaturen«, welche den Anforderungen des SignaturG entsprechend zustande gekommen sind, zulässt.

49 BGBI. I, S. 876; vgl. hierzu Roßnagel, Das neue Recht elektronischer Signaturen, NJW 2001, S. $1817 \mathrm{ff}$. 
dass auf belastende Entscheidungen verzichtet wird ${ }^{50}$, oder dahingehend, dass begünstigende Entscheidungen ergehen können ${ }^{51}$. Ob eine belastende bzw. begünstigende Entscheidung ergehen kann oder muss bzw. ob sie unterbleiben kann oder muss, hängt in diesen Fällen jedenfalls zum Teil ${ }^{52}$ von den gleichen materiellen Maßstabsnormen ab, die auch den gesellschaftlichen Trägern der Selbstregulierung ein Mindestma $B$ an Orientierung vorgeben (Beispiele: Die Anforderungen an die Produktbeschaffenheit, die Anforderungen des allgemeinen Umweltrechts ${ }^{53}$ etc.). Der Bezug zur staatlichen Entscheidungsverantwortung besteht hier mithin in der Existenz und im Umfang des Überschneidungsbereichs von staatlicher und gesellschaftlicher Orientierung am identischen Maßstab. Freilich erschöpft sich die Orientierung der Träger der gesellschaftlichen Selbstregulierung hierin nicht und sie entwickeln regelmäßig (und erwartungsgemäß) eigene Ordnungsmuster jenseits des gesetzten Standards, durch Bildung eigener, bereichsspezifischer Standards und Verwirklichungskonzepte. Dieser Mehrwert tritt deutlich zutage bei einem kompletten Umweltmanagementsystem, das zwar auch am geltenden Umweltrecht orientiert ist, darüber hinaus aber betriebseigene Standards setzt und zukunftsorientierte Gestaltungskonzepte verwirklichen hilft.

50 So etwa im Bauordnungsrecht, im Rahmen der Eigenüberwachung im Umweltrecht sowie unter den Bedingungen des novellierten Öko-Audit-Rechts, in dem Vollzugserleichterungen vorgesehen sind (vgl. soeben 1); ferner im Anwendungsbereich des KWG in Gestalt des Verzichts auf nachträgliche bankenaufsichtsrechtliche MaBnahmen und im Produktsicherheitsrecht in Gestalt des (zwingenden) Verzichts auf die Errichtung weiterer Zulassungshürden. Werden die Anforderungen der jeweils einschlägigen materiellen Maßstabsnorm durch die privaten Instanzen verfehlt, so realisiert sich die staatliche Gewährleistungsverantwortung in Gestalt des Erlasses der jeweiligen belastenden Maßnahme.

51 So beim Erlass des vorhabenbezogenen Bebauungsplans oder bei der Erteilung der Bankbetriebserlaubnis nach ordnungsgemäßem Aufbau eines Risk-Management-Systems, ferner bei der Erteilung von Anlagengenehmigungen nach ordnungsgemäßer Erfüllung von Ermittlungspflichten jenseits der eigenen betrieblichen Sphäre im Recht der Umweltverträglichkeitsprüfung (näher hierzu Schmidt-Preuß, VVDStRL 56 [1997], S. 178 f.).

52 Die staatliche Entscheidung hängt regelmäßig von der Erfülung weiterer Voraussetzungen ab. So müssen etwa vor Erteilung der Bankbetriebserlaubnis nach $\S 32 \mathrm{Abs} .1$ und $33 \mathrm{Abs} .1$ Satz 2 KWG zahlreiche weitere Voraussetzungen erfüllt sein bzw. sind die Entscheidungen über den Erlass belastender Maßnahmen (etwa im Recht der Umweltüberwachung) in das Ermessen der zuständigen Behörde gestellt.

$53 \mathrm{Ob}$ die Beachtung des geltenden Umweltrechts überhaupt Bestandteil des Prüfungsmaßstabs der umweltgutachterlichen Prüfung ist, und ob es dem Umweltgutachter verwehrt ist, eine Gültigkeitserklärung abzugeben, wenn er von einem RechtsverstoB des betroffenen Unternehmens gegen geltendes Umweltrecht Kenntnis erlangt hat, war unter dem bisherigen Umweltauditgesetz umstritten (ausführlich hierza Kloepfer/Bröcker, Umweltaudit und Umweltrechtskonformität, UPR 2000, S. 335 ff.; Kämmerer [Fn. 44], S. 64 ff.); nach lnkrafttreten der neuen EMAS-Verordnung (Fn. 45) besteht ein Verbot der Abgabe der Gültigkeitserklärung bei Verstößen gegen das materielle Umweltrecht (vgl. Langerfeld, UPR 2001, S. 220; Knopp, NVwZ 2001,S. 1098). 
Nicht behandelt werden die folgenden Themenkomplexe, die zwar alle einen Bezug zur Gesamtthematik aufweisen, aus unterschiedlichen Gründen aber jeweils am Rande des thematischen Kerns angesiedelt sind. Dies gilt für die private (selbstregulative) Normsetzung, die schwierige Probleme im Verhältnis zu den staatlichen Rechtsquellen aufwirft; überdies spielt schon die ausschließlich administrative Normsetzung (ganz ohne Privatisierung) nicht zuletzt in verfahrensmäßiger Hinsicht eine Sonderrolle innerhalb des Verwaltungsrechts, der vorliegend nicht nachgegangen werden kann ${ }^{54}$. Wichtige Anwendungsbeispiele finden sich im Recht der technischen Normung, so auch auf der Ebene vor dem oben (B III 1 c) skizzierten operativen Alltag im Produktsicherheitsrecht ${ }^{55}$ sowie bei der Standardsetzung im Recht der Rechnungslegung ${ }^{56}$. Ebenfalls nicht behandelt werden können die in den Kontext der regulierten Selbstregulierung fallenden staatlich induzierten Selbstverpflichtungen der Wirtschaft (v.a.: Umweltvereinbarungen), welche sich zum überwiegenden Teil auf die Rechtsetzung beziehen und normvertretende $\mathrm{Ab}$ sprachen darstellen ${ }^{57}$. Ausgeschieden werden ferner Erscheinungsformen der regulierten Selbstregulierung in den Bereichen Rundfunk ${ }^{58}$ sowie Wissenschaft und Forschung ${ }^{59}$, da es sich infolge der primär grundrechtlichen Zuordnung auch der Aufgabenerfüllung in öffentlich-rechtlichen Organisationsformen von vomherein um Sonderbereiche handelt.

$54 \mathrm{Zu}$ den Problemen der privaten Normsetzung vgl. nur die Beiträge von Voelskow, Private Regierungen in der Techniksteuerung. Eine sozialwissenschaftliche Analyse der technischen Normung, 1996; Trute, Vom Obrigkeitsstaat zur Kooperation, in: Hendler u.a. (Hrsg.), Rückzug des Ordnungsrechts im Umweltschutz, 1999, S. 13 ff.

55 Erste Ansätze einer Dogmatik der regulierten Selbstregulierung bei Schmidt-Aßmann, DV 2001, Beiheft 4, S. 269.

56 Hierzu jüngst Schuppert/Bumke, Verfassungsrechtliche Grenzen privater Standardsetzung -Vorüberlegungen zu einer Theorie der Wahl rechtlicher Regelungsformen (Regulatory Choice), in: Kleindiek/Oehler (Hrsg.), Die Zukunft des deutschen Bilanzrechts, 2000, S. 72 ff.; Hommelhoff/Schwab, Staatsersetzende Privatgremien im Unternehmensrecht, in: Drenseck/Seer (Hrsg.), FS Kruse, 2001, S. 653 ff.

57 Näher ausgeführt bei Faber (Fn. 35), S. 219 f;; zu den sich hierbei stellenden Problemen vgl. dies., S. 177 ff.; Frenz, Selbstverpflichtungen der Wirtschaft, 2001.

58 Vgl. hierzu Rossen, Selbststeuerung im Rundfunk, ZUM 1994, S. 224; Holznagel, DV 2001, Beiheft 4, S. 81 .

59 Vgl. dazu nur Trute, Die Wissenschaft vom Verwaltungsrecht, DV 27 (1999), S. 301. 
C. Vergewisserung: Funktionen des Verwaltungsverfahrens(-rechts) bei staatlicher Erfüllungsverantwortung

\section{Bewirkungsfunktion und Schutzfunktion}

Die auf unterschiedlichen Legitimationsgrundlagen beruhenden und in vielfältigen Ausprägungen figurierenden Funktionen des Verfahrens im Verwaltungsrecht lassen sich mit Schoch in der Bewirkungsfunktion einerseits und der Schutzfunktion anderseits bündeln. Der Bewirkungsauftrag des Verfahrens äußert sich in dessen Beitrag zur Erfüllung der jeweils in Frage stehenden Verwaltungsaufgaben, d. h. vor allem bei der Anwendung des materiellen Rechts, aber auch bei der Erfüllung damit $u$. U. einhergehender Verwaltungsaufträge wie etwa die Gewährleistung von Akzeptanz, Wirtschaftlichkeit oder Effizien $z^{60}$. In dem Umfang, in dem sich die materiellrechtliche Determinierung des administrativen Handelns ausdehnt, ist das Wort von der »dienenden Funktion « des Verfahrens ${ }^{61}$ berechtigt; in dem Umfang, in dem Entscheidungsspielräume bestehen und die Steuerungskraft des materiellen Gesetzes nachlässt, erlangt das Verfahren zusätzliche Legitimation und trifft das weitere Wort von der »Richtigkeitsgewähr durch Verfahren $\aleph^{62} \mathrm{zu}$. Im Schutzauftrag schließlich sind all diejenigen Teilfunktionen zusammengefügt, die dem frühzeitigen, vom gerichtlichen Rechtsschutz abgesetzten Schutz der betroffenen Rechte und Interessen gewidmet sind ${ }^{63}$.

60 Schoch, DV 25 (1992), S. 21 ff., 25 ff., 29 ff.; ferner jüngst Laubinger, in: Verfahrensrecht (Fn. 15), S. 53 f.; vgl. auch die Ausführungen im allgemeinen Teil der Entwurfsbegründung zu dem am 1.1.1977 in Kraft getretenen Verwaltungsverfahrensgesetz des Bundes (BTDrucks. 7/910, S. 29).

61 Verwendet in der Gesetzesbegründung der Bundesregierung zu den heutigen $\$ \S 45,46$ VwVfG (BT-Drucks. 7/910, S. 65; krit. hiergegen Hill, Verfahren [Fn. 18], S. 201 ff.).

62 Vgl. hierzu und allgemein zum Verhältnis zum materiellen Recht BVerfGE 53, 30 (57 ff., 62 ff. [Mülheim-Kärlich]); Badura, Das Verwaltungsverfahren, in: Erichsen (Hrsg.), Allgemeines Verwaltungsrecht, 11 . Aufl. 1998, § 33 Rn. 1 ff.; Hufen (Fn. 16), Rn. 11 ff., 16; Schuppert, Verwaltungswissenschaft (Fn. 15), S. 790 f., 805 f.

63 Vgl. Wahl, Verwaltungsverfahren zwischen Verwaltungseffizienz und Rechtsschutzauftrag, VVDStRL 41 (1983), S. 151 (160 ff.); Schoch, DV 25 (1992), S. 25 ff.; Schuppert, Verwaltungswissenschaft (Fn. 15), S. 802 f. 


\section{Teil-Funktionen und einzelne Verfahrenselemente}

Die nachfolgende Auswahl einzelner, für besonders relevant zu erachtender ${ }^{64}$ Verfahrenselemente soll die Suche nach funktionalen Äquivalenten (E) vorbereiten und die eher allgemein gehaltene Fragestellung ganz im Sinne des verfolgten dogmatischen Anliegens spezifizieren helfen.

\section{Distanz}

Neutralität und Objektivität sind klassische Ziele verfahrensrechtlicher Bestimmungen, die in der Verpflichtung zu rechtsstaatlichem Handeln und zur Wahrung verfahrensmäßiger Gleichheit wurzeln. Die Elemente zur Verwirklichung dieser Teilfunktion sind in den $\S \S 20,21 \mathrm{VwVfG}$ kodifiziert, durch die die Verwaltung als Ganzes sowie der einzelne Amtswalter vor Interessenkonflikten bewahrt und die Bürger vor einer Beteiligung befangener Amtsträger geschützt werden, und zwar in allen Phasen des Verfahrens, d. h. von der Einleitung bis zur Entscheidung ${ }^{65}$.

\section{Sachaufklärung und Betroffenenbeteiligung}

Ein ganz wesentliches, jedoch schwer zu erreichendes Ziel des Verfahrensrechts besteht in der Ermöglichung einer möglichst vollständigen und zutreffenden Sachverhaltsermittlung als Voraussetzung korrekter Rechtsanwendung, u. U. auch im Interesse des Einzelnen ${ }^{66}$. Wichtige diesbezügliche Verfahrenselemente im »Prozess der Informationsgewinnung und -verarbeitung « sind neben dem Untersuchungsgrundsatz (vgl. § 24 VwVfG) und den Mitwirkungs- (vgl. v. a. $\S 26$ Abs. 2 VwVfG) und Beweisregeln - mit entsprechenden Ermittlungs- und Verwertungsbefugnissen - die verschiedenen Formen der Beteiligung Betroffener, etwa im Rahmen von Erörterungs- und Anhö-

64 Vergleichbare, wenngleich nicht identische Schwerpunktsetzungen bei Hoffmann-Riem, in: Öffentliches Recht und Privatrecht, S. 318 ff.; Trute, DVBl. 1996, S. 960 f.; Burgi, DV 33 (2000), S. 18. Nicht geleistet werden kann an dieser Stelle die gleichermaßen wichtige wie gebotene Einbeziehung des Datenschutzrechts in seiner Gestalt als Sonderverwaltungsverfahrensrecht.

65 Vgl. nur Hufen (Fn. 16), Rn. 67 ff. (mit zutreffender Einreihung in die »übergreifenden Verfahrensgrundsätze«); Wolff/Bachof/Stober, Verwaltungsrecht II (Fn. 18), § 59 Rn. 13 ff. Zu den verschiedenen Bedeutungsgehalten von »Unparteilichkeit«, Distanz bzw. Neutralität vgl. aktuell Fehling, Verwaltung zwischen Unparteilichkeit und Gestaltungsaufgabe, 2001, S. 4 ff. Ausprägungen sind neben der hier nicht thematisierten (vgl. sogleich C III) organisationsrechtlichen Thematik der institutionellen Unparteilichkeit die personell-individuelle Unparteilichkeit (Fehling, S. 198 ff.) und die Unparteilichkeit der Verfahrensgestaltung (Fehling, S. 302 ff.), deren Teilelemente herkömmlich aber anderen Verfahrensfunktionen zugeordnet werden (v.a. der auch nachfolgend separierten Sachaufklärung und der Anhörung).

66 Vgl. Hufen (Fn. 16), Rn. 117 ff.; Wolff/Bachof/Stober, Verwaltungsrecht II (Fn. 18), \$60 Rn. $28 \mathrm{ff}$. 
rungsterminen (z. B. nach $\S 73 \mathrm{VwVfG}$ ). Durch sie kann zugleich die erforderliche »Chancengerechtigkeit« bei der Berücksichtigung bzw. Durchsetzung von Interessen bewirkt werden ${ }^{67}$.

\section{Transparenz, Beteiligtenanhörung und Begrïndung}

Transparenz zielt auf die Einsehbarkeit und Vorhersehbarkeit des Verwaltungshandelns und äußert sich in Informationspflichten (vgl. z. B. § 25 Satz 2 VwVfG) ${ }^{68}$ und insbesondere in der Pflicht zur Anhörung der Beteiligten, denen unter bestimmten Voraussetzungen ein diesbezügliches Recht eingeräumt ist (vgl. § $28 \mathrm{VwVfG}$ ); gleichzeitig dient die Anhörung auch dem vorgenannten Ziel erfolgreicher Sachaufklärung ${ }^{69}$. Mit dem Transparenzziel einher geht das Ziel des Vertraulichkeitsschutzes, welches namentlich durch die Akteneinsichtsvorschrift des $\S 29 \mathrm{VwVfG}$ näher ausgestaltet wird ${ }^{70}$. Nach erfolgter Entscheidung bildet schließlich das in $\S 39 \mathrm{VwVfG}$ kodifizierte Begründungsgebot ein Element rechtsstaatlicher Transparenz bereits mit Blick auf die Erfolgsaussichten eines etwaigen Rechtsbehelfsverfahren ${ }^{71}$.

\section{Verfahren und Organisation}

Ungeachtet des bekannten Umstandes, dass zwischen Verwaltungsverfahren und Verwaltungsorganisation gleitende Übergänge, wenn nicht Überschneidungsbereiche bestehen und die phänomenologische Unterscheidung zwischen Aufbau und Ablauf keinesfalls trennscharf ist ${ }^{72}$, können eine Reihe von Verfahrensfunktionen bzw. Verfahrenselementen schwerpunktmäßig der Organisation, und andere Funktionen wie Elemente schwerpunktmäßig dem Verfahren zugeordnet werden. So gehören Aspekte wie Pluralität, Kontrolle bzw. Aufsicht (u.a. mit diesbezüglichen Aufzeichnungspflichten) nicht in den Zusammenhang des vorgegebenen Themas, wenngleich in den bisherigen Untersuchungen zur Situation der formellen Anforderungen nach Privatisierung zwischen dem »Privatorganisationsrecht« und dem »Privatverfahrensrecht « nicht getrennt worden ist ${ }^{73}$.

$67 \mathrm{Zu}$ diesem Teil-Ziel und seiner Relevanz in selbstregulativen Kontexten vgl. HoffmannRiem, in: Öffentliches Recht (Fn. 2), S. 319 f.; vgl. ferner Pietzcker, Das Verwaltungsverfahren zwischen Verwaltungseffizienz und Rechtsschutzauftrag, VVDStRL 41 (1983), S. 204; Pitschas (Fn. 26), S. 729.

68 Vgl. Hufen (Fn. 16), Rn. 52; Wolff/Bachof/Stober, Verwaltungsrecht II (Fn. 18), §59 Rn. $5 \mathrm{f}$.

69 Vgl. WolffiBachof/Stober, Verwaltungsrecht II (Fn. 18), § 60 Rn. 54 ff.

70 Vgl. näher hierzu Wolff/Bachof/Stober, Verwaltungsrecht II (Fn. 18), §60 Rn. 90 ff.

71 Vgl. Hufen (Fn. 18), Rn. 294 ff.

72 Vgl. nur Schmidt-Aßmann, in: HdbStR III (Fn. 18), § 70 Rn. 7.

73 Etwa bei Hoffmann-Riem, in: Öffentliches Recht (Fn. 2), S. 318 ff.; Ritter, in: Verwaltungsorganisationsrecht (Fn. 8), S. 233 f., 235 ff.; Burgi, DV 33 (2000), S. 183 ff. 
Die zentralen verfassungsrechtlichen Anforderungen an das Verwaltungsverfahren ergeben sich aus einer Zusammenschau von Rechtsstaats- und Demokratieprinzip, den Grundrechten, insbesondere Art. 19 Abs. 4 GG und das Schutzpflichtkonzept, sowie aus den anderen Staatszielbestimmungen und den föderalen Strukturprinzipien ${ }^{74}$. Die meisten Verfahrenselemente lassen sich auf mindestens zwei Verfassungsgrundlagen zurückführen, so etwa die Befangenheitsregeln, welche primär im »Amt «, als Garanten der in personellorganisatorischer Hinsicht gebotenen Legitimation wurzeln, daneben aber auch rechtsstaatlich, im Erfordernis der Neutralität und Objektivität des Verwaltungshandelns, fundiert $\operatorname{sind}^{75}$. Das verfassungsrechtliche Normprogramm in Sachen »Verfahren « besteht aus Impulsen und Mindeststandards, die auf der Ebene des einfachen Rechts und der Rechtsdogmatik aufgenommen und weiterentwickelt worden $\operatorname{sind}^{76}$, weswegen nicht jede Ausprägung und Verästelung in den Verfahrensgesetzen als verfassungsgeboten angesehen werden darf.

D. Funktionsanalyse nach Privatisierung: Funktionale Verfahrens-Äquivalenz als Notwendigkeit und Option

\section{Fragestellung}

Seit Beginn der theoretischen Durchdringung des Kooperationsspektrums zwischen Staat und Gesellschaft wurde immer wieder die Frage nach der Notwendigkeit eines sog. Privatverfahrensrechts gestellt und vielfach sogleich dahingehend beantwortet, dass es einem »Gebot der funktionalen Äquivalenz« entspreche, die Rücknahme der staatlichen Erfüllungsverantwortung (auch) durch verfahrensrechtliche Instrumente des Öffentlichen Rechts und/

74 Vgl. zu den Einzelheiten Schmidt-Aßmann, in: HdbStR III (Fn. 18), § 70 Rn. 12 ff.; Ehlers, Verwaltung und Verwaltungsrecht im demokratischen und sozialen Rechtsstaat, in: Allgemeines Verwaltungsrecht (Fn. 62), § 4 Rn. 6 ff.; Burgi, DV 33 (2000), S. 190 ff. m.w.N.

75 Vgl. Isensee, Gemeinwohl und Staatsaufgaben im Verfassungsstaat, in: HdbStR III (Fn. 18), $\S 57$ Rn. 60 ff.; Dreier, in: ders. (Hrsg.), GG, Band 2, 1998, Art. 20 D Rn. 113 ff., 124 ff., sowie Schulze-Fielitz, aaO, Art. 20 R Rn. 190 ff.; ferner Burgi, DV 33 (2000), S. 191 m.w.N. Eingehend zu den demokratierechtlichen Anforderungen an »Public Private Partnerships $\ll$ Mehde, Ausübung von Staatsgewalt und Public Private Partnership, VerwArch 91 (2000), S. 540.

76 Burgi, DV 33 (2000), S. 190 m.w.N. 
oder des Privatrechts auszugleichen ${ }^{77}$. Der mir erteilte Auftrag besteht darin, jene Fragestellung zu präzisieren und anzugeben, unter welchen Voraussetzungen die behauptete Notwendigkeit entsteht und wie weit sie reicht. Dabei kann an die bereits erarbeitete (B II u. III) systematische Untergliederung des Gesamtspektrums angeknüpft werden.

»Funktionale Äquivalenz « ${ }^{78}$ bedeutet im vorliegenden Zusammenhang die Gewährleistung der soeben (C) skizzierten Sicherungs- und Schutzfunktionen des Verfahrens unter veränderten Rahmenbedingungen und unter Berücksichtigung des politisch erwünschten Hinzutretens der gesellschaftlichen Handlungsrationalität. In der nunmehr erreichten dogmatischen Phase kann nicht einfach von der Verantwortungsverschiebung auf die Notwendigkeit funktionaler Äquivalenz geschlossen werden. Vielmehr müssen die Veränderungen der Realvorgänge analysiert und zu dem jeweiligen Privatisierungskonzept in Beziehung gesetzt werden. Dabei wird sich zeigen, dass nicht durchgehend eine Notwendigkeit zur Herstellung funktionaler Äquivalenz besteht, diese aber zumindest stets eine beachtliche Option bildet. Erst im Anschluss an diese Funktionsanalyse können dann im folgenden Abschnitt (E) die einzelnen Verfahrenselemente als »funktionale Äquivalente« neu entwickelt werden. Bewusst wird nicht von einem Gebot der funktionalen Äquivalenz gesprochen, weil es (zunächst) nicht um eine (Verfassungs-)Rechtspflicht und auch nicht um eine dogmatische Figur geht, sondern um das Ergebnis einer Analyse der Ordnungsidee »Verfahren« nach Privatisierung.

\section{Verwaltungshilfe: Private Vorbereitung staatlicher Entscheidungen}

In den hier relevanten Konstellationen der Verwaltungshilfe (B II) werden Verwaltungsentscheidungen durch private Träger vorbereitet, worin sich der private Beitrag - sei er planerischen, sachverständig-beratenden oder verfah-

77 So Hoffmann-Riem, Verfahrensprivatisierung als Modernisierung, DVBI. 1996, S. 230; Trute, in: Verantwortungsteilung (Fn. 5), S. 27 f. Dabei bleibt ebenso wie etwa in den Beiträgen von Lübbe-Wolf/Steenken, Privatisierung umweltbehördlicher Aufgaben, ZUR 1993, S. 263 (268 ff.); Reinhardt, Die Uberwachung durch Private im Umwelt- und Technikrecht, AöR 118 (1993), S. 624, oder auch Hoffimann-Riem, in: Öffentliches Recht (Fn. 2), S. 318 ff., sowie Ritter, in: Verwaltungsorganisationsrecht (Fn. 8), S. 235 ff., offen, ob es sich um eine Rechtspflicht oder um das Ergebnis einer Funktionsanalyse handelt. Als »Prinzip der funktionalen Äquivalenz« tauchen die dort entfalteten Überlegungen unter A (»Allgemeines $(1)$ in der Begründung des am 19.9.2001 vom Bundeskabinett angenommenen Entwurfs einer EMAS-Privilegierungsverordnung (Fn, 45) auf.

78 Dahinter verbirgt sich eine Begriffsanleihe bei Luhmann, Zweckbegriff und Systemrationalität (1968), 1973, S. 168 f., 236 ff.; zur Verwendbarkeit dieses Begriffs in analytischen bzw. dogmatischen Kontexten vgl. Schulze-Fielitz, Zusammenspiel von öffentlich-rechtlichen Kontrollen der Verwaltung, in: Schmidt-ABmann/Hoffmann-Riem (Hrsg.), Verwaltungskontrolle, 2001, S. 291 (302 f.). 
renstechnisch-projektmanagenden - Charakters auch erschöpft. Die Analyse des Realvorgangs erweist, dass zwar die Entscheidungsgewalt äußerlich betrachtet beim Staat verblieben ist, dass aber vielfach nur noch das ratifiziert werden kann, was die private Entscheidungsvorbereitung ergeben hat, sei es, aufgrund geringerer behördlicher Sachkompetenz, sei es aufgrund mangelnder Verwaltungskapazität, sei es wegen der Komplexität der betreffenden Aufgabe oder der Situationsbezogenheit und Unwiederholbarkeit der relevanten Abläufe ${ }^{79}$.

Würde man nun die verfahrensrechtlichen Anforderungen, d. h. den Einsatz der oben beschriebenen Verfahrenselemente, ausschließlich an das Vorliegen einer staatlichen Entscheidung knüpfen, gingen sie vielfach ins Leere und die Bewirkungsfunktion des Verfahrensrechts könnte nicht, zumindest nicht vollständig, erfüllt werden. Hinzu kommt, dass private Vorbereitungsbeiträge häufig gerade im Zusammenhang mit solchen Aufgaben erbracht werden, die durch das Bestehen weiter Entscheidungsspielräume gekennzeichnet sind, und dass die privaten Träger überdies, bewusst oder unbewusst, in geringerem $\mathrm{Maße}$ an materielle Vorgaben gebunden werden. Unter diesen Umständen kommt den formalen Sicherungen eine um so größere Bedeutung im Hinblick auf die Richtigkeit der (dann vom Staat) zu treffenden Entscheidung $\mathrm{zu}^{80}$. Aber nicht nur die Bewirkungsfunktion, auch die Schutzfunktion des Verfahrensrechts ist herausgefordert. Unterbleibt z.B. die Anhörung eines Beteiligten im Stadium der privaten Entscheidungsvorbereitung, kann es sein, dass die hierdurch vermittelten Interessen oder gar Rechte im Rahmen der abschließenden Verwaltungsentscheidung zu spät oder überhaupt nicht berücksichtigt werden. In den Fällen der Vorbereitung staatlicher Entscheidungen durch Verwaltungshelfer ist somit die Herstellung funktionaler Äquivalenz in verfahrensmäßiger Hinsicht notwendig.

79 Zur Analyse des Realvorgangs und des diesbezüglichen Gefährdungspotenzials: Brohm, in: HdbStR II (Fn. 33), \& 36 Rn. 31 f.; Schneider, Nachvoliziehende Amtsermittlung bei der Umweltverträglichkeitsprüfung, 1991, S. $186 \mathrm{f} ; \mathrm{Wahl}$, in: Verwaltungsorganisationsrecht (Fn. 3), S. 306 f.; Pietzcker, in: Verfahrensprivatisierung (Fn. 67), S. 284 ff.; HoffmannRiem, DVB1. 1996, S. 225 (229); Di Fabio, VVDStRL 56 (1997), S. 267 f.; Huber, DVB1. 1999, S. 489 (492, 494 f.); Burgi, DV 33 (2000), S. 192 ff. Klassisch: Forsthoff, Lehrbuch des Verwaltungsrechts, Erster Band, Allgemeiner Teil, 10. Aufl. 1973, S. 76.

80 Explizit (zur »Verfahrensprivatisierung«): Hoffmann-Riem, in: Öffentliches Recht (Fn. 2), S. 318; Schuppert, Verwaltungswissenschaft (Fn. 15), S. 813. 
1. Bezug zu staatlichen Entscheidungen (Identität des materiellen Maßstabes)

Wenn und soweit ein Träger gesellschaftlicher Selbstregulierung und eine staatliche Verwaltungsstelle zur Orientierung an den gleichen materiellen Maßstäben verpflichtet sind und die staatliche Verwaltungsstelle auf die Ergebnisse der selbstregulierenden Tätigkeit mit dem Erlass einer begünstigenden (etwa: Erteilung einer Bankbetriebserlaubnis) bzw. mit dem Unterlassen einer nachteiligen Entscheidung (z. B. im Bereich der Marktüberwachung) reagieren soll (vgl. B III 2), dann muss sie jene Ergebnisse akzeptieren können $^{81}$. Das aber kann sie nur, wenn es Richtigkeitsgaranten gibt und einer der Richtigkeitsgaranten ist das Verfahren zur Gewinnung von Ergebnissen, was oben (C I) mit dem Begriff der Bewirkungsfunktion umschrieben worden ist. Ihr zur Seite tritt wiederum die Schutzfunktion, der es um die Wahrung der auch hier betroffenen Rechte und Interessen zu tun ist. Diese Zusammenhänge lassen sich besonders prägnant am Beispiel der EMAS-Privilegierungsverordnung (Öko-Audit) veranschaulichen: Weil die betroffenen Unternehmen am Öko-Audit teilgenommen haben und damit die Einhaltung der materiellen Umweltstandards dokumentiert ist, können ihnen durch die zuständige Behörde Erleichterungen bei der nachträglichen Umweltüberwachung gewährt werden - wenn das Ergebnis der materiellen Umweltrechtskonformität in einem Öko-Audit-Verfahren mit Richtigkeits- und Schutzgewähr gewonnen worden ist ${ }^{82}$. Die Herstellung funktionaler Verfahrensäquivalenz ist also insoweit, in Anbetracht des skizzierten Kondominiums, notwendig.

Sie ist nicht notwendig außerhalb des Kondominialbereichs, d. h. soweit sich mit der Selbstregulierung ein Mehrwert im oben (B III 2) beschriebenen Sinne verbindet. Denn in Ermangelung eines Bezugs zu einem materiellen Verwaltungsauftrag entsteht die Bewirkungsfunktion des Verfahrensrechts nicht und die Schutzfunktion geht ebenfalls ins Leere, weil staatlich geschützte Interessen und Rechte von Betroffenen insoweit nicht beeinträchtigt sind. Die Herstellung funktionaler Verfahrens-Äquivalenz ist in diesem Umfang mithin lediglich eine Option, die aber teilweise bereits dadurch eingelöst worden ist, dass in Anbetracht des Überschneidungsbereichs der materiellen Maßstäbe

81 Hufen (Fn. 16), Rn. 6, stellt darauf ab, dass der Staat wdie Verantwortung für die Ergebnisse der Selbstregulierung « übernimmt. Auf die materiellen Anforderungen hebt auch Hoffmann-Riem, DVB1. 1996, S. 228 f., ab.

82 Explizit zum Gedanken der funktionalen Verfahrens-Äquivalenz im Öko-Audit-Recht: Laskowski, in: Verantwortungsteilung (Fn. 5), S. 101 f.; Knopp, NVwZ 2001, S. 1098. 
funktionale Verfahrensäquivalente geschaffen worden sind. Um wiederum mit einem Beispiel zu sprechen: Müssen infolge der teilweisen Neu-Orientierung des Öko-Audits am Maßstab des materiellen Umweltrechts und der Schaffung von Privilegierungen Befangenheitsregeln für die Umweltgutachter etabliert werden, dann "profitieren « von deren persönlichen Qualitäten auch die eigentlich überschneidungsfreien Bereiche der betrieblichen Umweltpolitik.

\section{Fehlender Bezug zu staatlichen Entscheidungen}

Dort schließlich, wo überhaupt kein Bezug zu staatlichen Entscheidungen durch die gemeinsame Orientierung an einem materiellen Maßstab existiert, besteht auch keine Notwendigkeit zur Herstellung funktionaler Äquivalenz. Im gemeinsamen Ordnungskonzept ist kein materieller Verwaltungsauftrag vorgesehen, vielmehr liegen Programmaufstellung und -verwirklichung ganz in gesellschaftlicher Hand. Die dort vorgesehenen Vorgänge der Informationsgewinnung und -verarbeitung im Hinblick auf eine privat getroffene Entscheidung (etwa die des Umweltgutachters bisheriger Prägung) stellen Privatverfahren dar. Es ist gerade Ausfluss des gemeinsamen Ordnungskonzepts, sie nicht als ein funktionales Äquivalent, sondern als Verfahren auf der Basis privater Handlungsrationalität ablaufen zu lassen. Ändern sich die Gegebenheiten und wird eine bislang nicht bestehende Verzahnung mit einem Verwaltungsauftrag herbeigeführt, dann bringt dies auch die Entstehung der Notwendigkeit funktionaler Äquivalenz mit sich - wie soeben mit Blick auf die bevorstehenden Veränderungen des Öko-Audit-Konzepts geschildert. Interessanterweise erfolgen diese übrigens mit dem Ziel, die Attraktivität des Ordnungskonzeptes zu steigern und die Zahl der Teilnehmer zu erhöhen ${ }^{83}$.

Dass keine Notwendigkeit der Herstellung funktionaler Äquivalenz besteht, heißt wiederum nicht, dass einzelne Verfahrenselemente, die die beschriebenen Sicherungs- und Schutzfunktionen erfüllen helfen, nicht auch hier zum Einsatz gelangen können, wenn der Gesetzgeber und/oder die das Ordnungskonzept tragenden Kräfte sich davon Vorzüge versprechen. Aus dieser Perspektive geben die nachfolgenden Ausführungen Aufschluss über Optionen, nicht über Pflichten und funktionale Äquivalente. 
Ging es bislang um die Notwendigkeit der Herstellung funktionaler Verfahrens-Äquivalenz, so ist nunmehr zu klären, wie die Zurverfügungstellung der funktionalen Äquivalente erfolgen kann und wie diese aussehen können. Das Recht ist in dieser Situation zur Bereitstellung der erforderlichen Gemeinwohlsicherungen nach Verantwortungsteilung bzw. Privatisierung berufen ${ }^{84}$, und zwar als Recht der Regulierung (vgl. A II). Selbstverständlich bilden die Verfahrensaspekte nicht den einzigen Gegenstand der Regulierung, sondern sie treten neben die jeweils relevanten materiellen Fragen und neben die organisatorischen Aspekte (vgl. III) im Anschluss an eine Verantwortungsteilung. Die hier gewonnenen Erkenntnisse verstehen sich daher als Teil eines (im Bereich der regulierten Selbstregulierung noch zu entwerfenden [vgl. bereits B III 1]) Gesamtkonzepts der Regulierung.

\section{Strukturschaffungspflicht statt unmittelbare (verfassungs-)verfahrensge- setzliche Bindung}

Weder die Verwaltungshelfer noch die Träger einer Selbstregulierung sind unmittelbar an die oben (C IV) skizzierten Vorgaben des Verfahrensverfassungsrechts gebunden ${ }^{85}$ und ihr Verhalten unterfällt grundsätzlich auch nicht den an die »Behörden « adressierten Verfahrensnormen des VwVfG ( $\S 1$ Abs. 1 u. 4$)^{86}$. Es ist daher Sache des Staates, allein bzw. im Zusammenwirken mit den jeweiligen gesellschaftlichen Kräften die notwendigen funktionalen Äquivalente auf der normativen Ebene des einfachen Rechts und/oder auf der Ebene administrativer Regulierung zu schaffen. Da er im Hinblick auf die organisatorischen Aspekte nach Verantwortungsteilung vor einer vergleich-

84 Zur Bereitstellungsfunktion des Rechts im Kontext von Verantwortungsteilung und Privatisierung vgl. Schuppert, Kooperationsverhältnisse (Fn. 12), S. 67 ff. m.w.N.

85 Dies gilt namentlich für das Demokratieprinzip, welches nach Wortlaut und Systematik der Art. 20 Abs. 1 u. 2 GG, 21 Abs. 1 GG ein Bauprinzip des Staates, kein Strukturprinzip der Gesellschaft bildet (vgl. nur BVerfGE 83, 60 [72]; BVerfG, DVBI. 1995, S. 1291; Stern, Staatsrecht I, 2. Aufl. 1984, S. 629 ff.) und für das Rechtsstaatsprinzip, dessen Staatsbezogenheit als Grundentscheidung über die Staatsordnung noch evidenter ist (vgl. bereits Burgi, DV 33 [2000], S. 193). Gegen eine Grundrechtsbindung in diesen Zusammenhängen Trute, in: Schuppert, Verantwortungsteilung (Fn. 5), S. 42 f.; ausführlich zur grundrechtlichen Situation bei der Verwaltungshilfe vgl. Burgi (Fn. 13), S. $328 \mathrm{ff}$.

86 Unter bestimmten, bei Burgi, DV 33 (2000), S. 20, geschilderten Umständen, ist ausnahmsweise die Erfassung privater Träger, die »im Rahmen eines Verwaltungsverfahrens tätig werden«, gemäß $\S 26$ Abs. 1 Ziffer 2 VwVfG i.V.m. $\S \S 20$ Abs. 1, 21 Abs. 1 VwVfG möglich. 
baren Herausforderung steht und es hier wie dort um die Herausbildung formaler Ordnungsstrukturen geht, sei empfohlen, zur Kennzeichnung von Ausgangslage und rechtlichem Horizont den bereits anderenorts entfalteten Begriff der »Strukturschaffungspflicht $\varkappa^{87} \mathrm{zu}$ verwenden. Er bringt zum Ausdruck, dass im Hinblick auf die Formalanforderungen eine Abweichung vom Normal- und Regelfall staatlicher Entscheidungsverantwortung vorliegt und eine aufgabenspezifische Berücksichtigung der veränderten Rahmenbedingungen sowie der privaten Handlungsrationalität indiziert ist.

Mit dem Rechtsinstitut der Strukturschaffungspflicht verbindet sich $\mathrm{m}$. E. zweierlei: Erstens, dass es nicht um die Übertragung der im Falle staatlicher Entscheidungsverantwortung bestehenden verfahrensbezogenen Anforderungen im Verhältnis eins zu eins geht, sondern dass die Schaffung andersartiger, spezifischer Strukturen vonnöten ist. Zweitens, dass es nicht nur um das Verfahren im Bereich des involvierten staatlichen Trägers, sondern auch um die formalen Strukturen im Hinblick auf das Tätigwerden von staatsexternen Einheiten geht.

Im Hinblick auf die Verwaltungshilfe, bei der es ja um ein unmittelbar staatsaufgabenbezogenes Tätigwerden geht (vgl. B II), konnte in einer früheren Untersuchung ${ }^{88}$ bereits nachgewiesen werden, dass die Strukturschaffungspflicht verfassungsrechtlich verankert ist, weil die abgespaltenen Vorbereitungsbeiträge gezielt verselbständigt worden sind und die unverändert von einem Amtsträger getroffene Entscheidung als verfassungsrechtlich relevantes »Endprodukt« legitimatorische Vorwirkungen ${ }^{89}$ zeitigt; die Verfassung duldet nicht den realitätsfernen Trost, dass die Letztentscheidung ja bei einem Amtsträger liege und daher legitimierbar $\mathrm{se}^{90}$, sondern sie fordert, die verfahrensrechtlichen Strukturen dort zu schaffen, wo ein Teil des Verfahrens ab-

87 Zunächst entwickelt bei Burgi, Funktionale Privatisienung (Fn. 13), S. 378 ff., im Hinblick auf die Verwaltungshilfe, und sodann weiterentwickelt durch dens., DV 33 (2000), S. $200 \mathrm{ff}$., generell im Hinblick auf die Erscheinungsformen der privaten Vorbereitung staatlicher Entscheidungen.

88 Burgi, DV 33 (2000), S. 200 ff. Die dortigen Ausführungen erstrecken sich auch auf die Erscheinungsform der Aufstellung eines Vorhaben- und Erschließungsplans im Gesamtbereich der regulierten Selbstregulierung (S. 203), weil bei dieser die für den Fall der Verwaltungshilfe durch Erbringung vorbereitender Teilbeiträge aufgestellten Anforderungen als erfüllt anzusehen sind.

89 Der Ansatz, die »Legitimationsverantwortung « des Staates über die Einwirkung auf die von ihm ins Spiel gebrachten privaten Akteure zu entfalten, ist bereits zuvor entwickelt worden von Trute, DVBI. 1996, S. 954 f. (960 f.), und dems., in: Verwaltungsorganisationsrecht (Fn. 3), S. 270 ff, 290 ff.; vgl. ferner Schmidt-Preußs, VVDStRL 56 (1995), S. 203 ff.; Röhl, DV 29 (1996), S. 401 ff., und Möllers, VerwArch 90 (1999), S. 197 ff.

90 Mit dieser Tendenz aber Oebbecke, Weisungs- und unterrichtungsfreie Răume in der Verwaltung, 1986, S. 79 ff.; Emde, Die demokratische Legitimation der funktionalen Selbstverwaltung, 1991, S. 215, sowie in neuerer Zeit und speziell im Hinblick auf die Einbeziehung von Sachverständigen in der Vorbereitungsphase Jestaedt, Demokratieprinzip und Kondominialverwaltung, 1993, S. 587 f., 590 ff. Krit. insoweit bereits Brohm, in: HdbStR II (Fn. $33), \S 36 \mathrm{Rn} .31 \mathrm{ff}$. 
läuft. Im Hinblick auf die regulierte Selbstregulierung, bei der es ja an einem Bezug zur Staatsaufgabe fehlt und die bislang dogmatisch noch nicht in dem Maße verfestigt worden ist, dass eine einheitliche verfassungsrechtliche Erfassung möglich wäre (vgl. B III), ist vorerst noch keine verfassungsrechtliche Fundierung der Strukturschaffungspflicht möglich. Das schließt nicht aus, dass die am Beispiel der Verwaltungshilfe entwickelten Voraussetzungen auch in bestimmten Konstellationen der regulierten Selbstregulierung erfüllt sein mögen, oder dass jenseits der demokratierechtlichen und rechtsstaatlichen Maßstabsnormen den grundrechtlichen Schutzpflichtbestimmungen ${ }^{91}$ Pflichten zur Ausgestaltung von Organisation und Verfahren entnommen werden können. An dieser Stelle kann etwaigen verfassungsrechtlichen Grundlagen freilich nicht nachgegangen werden, vielmehr gilt es, die sachlich vorrangige Aufgabe der Strukturierung der Verfahrensrechtsverhältnisse und der Beschreibung der einzelnen Verfahrenselemente in Angriff zu nehmen.

\section{Verfahrensrechtsverhältnisse nach Privatisierung}

Zur Kennzeichnung der potentiellen Rechtsbeziehungen verfahrensrechtlicher Art soll der bereits etablierte Begriff des Verfahrensrechtsverhältnisses Verwendung finden, der allgemein die heuristische Funktion erfüllt, das »Beziehungsgefüge « zwischen den Verfahrenssubjekten zu strukturieren ${ }^{92}$. Im Kontext der Verantwortungsteilung, in dem man es stets mit mehrpoligen Interessenlagen zu tun hat, ermöglicht die Perspektive des Verfahrensrechtsverhältnisses die Erfassung und Offenlegung jener Interessenlagen, zugleich kann die Zuordnung einzelner Verfahrenselemente vorbereitet werden. Es liegt auf der Hand, Funktion und Inhalt einzelner Verfahrenselemente in Abhängigkeit von den unterschiedlichen Interessen- und Verfahrensstrukturen zu bestimmen. Dabei wird deutlich werden, dass mit einer Privatisierung die Zahl der Verfahrensrechtsverhältnisse typischerweise wächst.

Der Unterscheidung der potentiellen Verfahrenrechtsverhältnisse muss die Benennung der beteiligten Subjekte vorangehen: Bei der Verwaltungshilfe setzt sich das Beziehungsgefüge zusammen aus den beteiligten staatlichen Stellen, dem privaten Verwaltungshelfer und den von der Aufgabenwahrnehmung betroffenen Dritten, wobei zum Kreis der Dritten je nach den gegebenen faktischen und normativen Umständen rechnen können Nachbarn

91 Explizit jetzt Schmidt-Aßmann, DV 2001, Beiheft 4, S. 267 f.

92 Nach Martens, Die Praxis des Verwaltungsverfahrens, 1985, S. 41 ff; Hill (Fn. 18), S. 271 ff.; Schmidt-Aßmann, in: HdbStR III (Fn. 18), $\$ 70$ Rn. 31; Stelkens/Schmitz, in: Stelkens/Bonk/Sachs, VwVfG (Fn. 20), § 9 Rn. 5 ff. Zu den Möglichkeiten und Grenzen eines Denkens in Rechtsverhäl tnissen im Verwaltungsrecht vgl. allgemein von Danwitz, Zu Funktion und Bedeutung der Rechtsverhältnislehre, DV 30 (1997), S. 339 (350 ff.). 
(i.w.S.), Destinatäre, Konkurrenten etc. Bei der regulierten Selbstregulierung ist das Beziehungsgefüge regelmäßig komplexer. Neben den staatlichen Stellen agieren diejenigen privaten Kräfte, die die Selbstregulierung tragen. Anders als bei der Verwaltungshilfe wurzelt ihr Tätigwerden in der Selbstbetroffenheit, d. h. bei einer Gemeinwohlverwirklichung in der klassischen Form staatlicher Erfüllungsverantwortung würden sie zum Kreis der von der Aufgabenerfüllung Betroffenen, zu den Adressaten staatlicher Verfügungen gehören. Mit der Schaffung eines gemeinsamen Ordnungskonzepts werden sie (auch) zu Trägern der Gemeinwohlverwirklichung, freilich ohne die Betroffeneneigenschaft abstreifen zu können. Sie teilen diese weiterhin (und wiederum) mit betroffenen Nachbarn, Destinatären, Konkurrenten etc. ${ }^{93}$. Nun gibt es aber auch Ordnungskonzepte gesellschaftlicher Selbstregulierung, bei denen die Trägerseite der Selbstregulierung durch professionelle Dritte als "private Implementationsinstanzen $"$ " ${ }^{94}$ verstärkt wird. Für diese bildet die Selbstregulierung regelmäßig den Gegenstand ihrer grundrechtlichen Berufsund Geschäftstätigkeit, ebenso wie bei den Verwaltungshelfern, die stets Nicht-Aufgabenbetroffene sind. Wichtige Beispiele privater Implementationsinstanzen bilden die Betriebsbeauftragten, die Öko-Audit-Gutachter, die Benannten Stellen im Produktsicherheitsrecht und die anerkannten Prüf- und Bestätigungsstellen nach dem SignaturG.

\section{Staat-Dritte}

Im Verhältnis zwischen den staatlichen Stellen und den betroffenen Dritten stellt sich die Lage wie folgt dar: Bei der Verwaltungshilfe bleibt die staatliche Verwaltung Trägerin des Verwaltungsverfahrens und trifft die abschließenden Entscheidungen, jeweils unter Anwendung der hierfür geltenden verfahrensrechtlichen Bestimmungen (etwa der $\S \S 20,21$ oder $28 \mathrm{VwVfG}$ ). Da aber ein Teil der materiellen Entscheidungsgewalt auf den Vorbereitungshelfer übergegangen ist (vgl. D II), lautet die entscheidende Frage, wie die notwendige funktionale Verfahrens-Äquivalenz innerhalb der entstandenen weiteren Beziehungen (vgl. 2 und 3) bewirkt werden kann. Diese Frage stellt sich erst recht bei der regulierten Selbstregulierung, wo die Notwendigkeit funktionaler Verfahrens-Äquivalenz gerade deswegen entstanden ist (vgl. D III), weil

93 Schmidt-Aßmann, DV 2001, Beiheft 4, S. 262, unterscheidet zwischen $» D r i t t e n «$, worunter vor allem die "Destinatäre von Selbstregulierungseinrichtungen« zu verstehen seien, den »Teilnehmern eines Selbstregulienungssystems «, den »Konkurrenten« und den »Betroffenen« (wohl: im Übrigen).

94 Begriff nach Schmidt-Aßmann, DV 2001, Beiheft 4, S. 259, der die Implementationsinstanzen innerhalb der Selbstregulierung von den »staatlichen Implementationsinstanzen « unterscheidet, wonunter die zur Regulierung berufenen Verwaltungsbehörden und sonstige Verwaltungsstellen fallen sollen. 
erst gar kein Verfahrensrechtsverhältnis mit einer staatlichen Stelle besteht, die betroffenen Dritten vielmehr auf einen privaten Selbstregulierungsträger bzw. die eingeschaltete private Implementationsinstanz angewiesen sind. In dem Umfang, in dem staatliche Stellen noch tätig werden, etwa beim Erlass von Umweltüberwachungsmaßnahmen gegenüber einem auditierten Unternehmen, ändert sich an ihrer Verfahrensbeziehung zu den betroffenen Nachbarn und Konkurrenten nichts.

\section{Staat-private Träger (ggf. mit privater Implementationsinstanz)}

Verfahrensbeziehungen zwischen staatlichen Stellen und den privaten Trägern entstehen, wenn sich die Regulierung nicht auf der normativen Ebene erschöpft, sondern administrativ fixierte Kooperationsbeziehungen bestehen. Dies ist durchgehend der Fall bei der Verwaltungshilfe, die auf einem in der Regel vertraglichen Veranlassungsakt beruht, welcher sich an einen Vorgang der »Informationsgewinnung und -verarbeitung «, also an ein Verfahren, anschließ $\mathrm{t}$; gleiches gilt für spätere Veränderungen. Bei der regulierten Selbstregulierung entsteht eine vergleichbare Beziehung zwischen dem Staat und den Kräften der Selbstregulierung zum ersten dann, wenn die Verwaltung mit begünstigenden oder belastenden Maßnahmen - also mit einem Verzahnungsakt - auf die Ergebnisse einer Selbstregulierung reagiert (etwa bei der Erteilung der Bankbetriebserlaubnis; vgl. im Weiteren B II 2) und zum zweiten (und vor allem) dann, wenn private Implementationsinstanzen eingeschaltet sind, die einer staatlichen Akkreditierung bedürfen, wie es bei den Öko-Audit-Gutachtern, den Benannten Stellen oder bei den anerkannten Prüf- und Bestätigungsstellen nach dem SignaturG der Fall ist.

\section{Private Träger (ggf. mit privater Implementationsinstanz) - Dritte}

Der letzte Blick gilt dem Beziehungsgefüge zwischen dem privaten Träger bzw. den privaten Implementationsinstanzen und den betroffenen Dritten, wobei bei der Einschaltung einer privaten Implementationsinstanz noch zuvor ein Verfahrensrechtsverhältnis gegenüber den die Selbstregulierung tragenden privaten Kräfte entsteht. Das Tätigwerden von Verwaltungshelfern (etwa von Verfahrensmanagern) kann ebenso drittrelevant sein wie das Handeln von privaten Selbstregulierungsträgern (Beispiel: Verhältnis Bank - Anleger) bzw. von privaten Implementationsinstanzen, vom Betriebsbeauftragten, der mit den Nachbarn verhandelt, bis zur Benannten Stelle, die den Verbraucherinteressen zu dienen hat. Besteht eine Drittrelevanz, ist ein Verfahrensrechtsverhältnis jedenfalls konstruierbar. 
Die nachfolgend untersuchten Verfahrenselemente bilden eine Auswahl, die thematisch an die bereits oben, in der Situation staatlicher Erfüllungsverantwortung (C II) präsentierte Zusammenstellung anknüpft. Freilich können die ausgewählten Elemente nicht erschöpfend, d. h. in ihrer Ausgestaltung in den einzelnen Rechtsbereichen der funktionalen Verwaltungshilfe und vor allem der regulierten Selbstregulierung behandelt werden. Worum es geht, ist die systematische Zuordnung bestehender bzw. denkbarer Verfahrenselemente zu den soeben skizzierten Verfahrensrechtsverhältnissen, nicht zuletzt mit Blick auf etwaige Defizite.

\section{Distanz}

Beim Thema »Distanz« offenbart sich zunächst die Flexibilität des hier vorgeschlagenen Instituts (E I) der Strukturschaffungspflicht (anstelle einer Übertragung der sonst geltenden Anforderungen im Verhältnis eins zu eins), und zwar in Anbetracht der regulierten Selbstregulierung. Da die dort intendierten Vorteile gerade durch die Heranziehung der Aufgabenbetroffenen, mithin von »befangenen « privaten Kräften (z.B. Investoren, umweltbelastende Betriebe, Hersteller sicherheitsrelevanter Produkte etc.), und zwar vermittels der Stärkung von deren Eigenverantwortung, erreicht werden sollen, darf die Etablierung verfahrensmäßiger Anforderungen nicht dazu führen, dass eben dieses ausgeschlossen wäre ${ }^{95}$. Das bedeutet, dass im Bereich der regulierten Selbstregulierung über Befangenheitsregeln erst nachzudenken ist, wenn zusätzlich private Implementationsinstanzen tätig sind, denen dann die notwendige Distanz abverlangt werden kann. Lässt sich allerdings einer gesetzlichen Privatisierungsvorschrift - konkret dem $\S 4 \mathrm{~b}$ BauGB (betreffend die Vorbereitung von Verfahrensschritten im Rahmen der Bauleitplanung) - nicht eindeutig entnehmen, ob nur »Dritte (im Sinne einer Verwaltungshilfe) oder auch Aufgabenbetroffene (im Sinne einer regulierten Selbstregulierung) herangezogen werden dürfen, reduziert die Notwendigkeit funktionaler Verfahrens-Äquivalenz den Auslegungsspielraum zugunsten der distanzwahrenderen Alternative der Verwaltungshilfe ${ }^{96}$.

95 Burgi, DV 33 (2000), S. 204. Einen umfassenden, teilweise an anderen Kriterien als dem hier zugrunde gelegten (Kriterium »Typus von Verantwortungsteilung () orientierten Gesamtüberblick gibt aktuell Fehling (Fn. 65), S. 351 ff., mit eigenen Ansätzen ab S. 430 ff.

96 So im Ergebnis auch Reidt, NVwZ 1998, S. 593; Battis/Krautzberger/Löhr, BauGB, 7. Aufl. 1999, § 4b Rn. 6; anders Wagner, Bauleitplanverfahren - Änderungen durch die BauGBNovelle, BauR 1997, S. 715 ff,; Schmidt-Eichstaedt, Der Dritte im Baugesetzbuch, BauR 1998, S. 899 (900 ff.). 
Distanzschutz nach Privatisierung ist zunächst ein Thema im Rahmen des eröffneten Verfahrensrechtsverhältnisses zwischen dem Staat und dem Verwaltungshelfer bzw. der privaten Implementationsinstanz. Mit der Veranlassung zur Verwaltungshilfe auf administrativem Wege entsteht ein Verfahrenrechtsverhältnis, bezogen auf den jeweiligen Veranlassungsakt, bei dessen Erlass befangene Amtsträger nicht mitwirken dürfen, was sich aus den $\S \S 20,21$ $\mathrm{VwVfG}^{97}$ bzw. aus $\S 16$ der Vergabeverordnung ${ }^{98}$ ergibt. Entsprechendes gilt (bei der regulierten Selbstregulierung) beim Erlass eines Verzahnungsaktes im oben, B III 2 u. E II 2, geschilderten Sinne sowie bei der administrativen Regulierung einer Implementationsinstanz (z.B. bei der Akkreditierung von Benannten Stellen).

Noch wichtiger für den Distanzschutz ist es, die Gelegenheit eines solchen administrativen Kontakts zu nutzen, um den Verwaltungshelfer bzw. die private Implementationsinstanz zu Neutralität und Objektivität in deren Verhältnis zu den betroffenen Dritten zu verpflichten. Das beginnt mit der Aufnahme dieser Anforderungen in die Leistungsbeschreibung vor der Auftragsvergabe an einen Verwaltungshelfer ${ }^{99}$ und setzt sich fort beim Inhalt des Veranlassungsvertrages, in dem eine Befangenheitsklausel nicht fehlen darf. Beide Aspekte werden in der Praxis vielfach nicht beachtet. Bei der regulierten Selbstregulierung bietet sich die Gelegenheit zur Gestaltung der Verhältnisse innerhalb der Selbstregulierung und zu den betroffenen Dritten aus Anlass eines Verzahnungsaktes, etwa, wenn die Bestellung eines zu intensiv in die Unternehmensleitung eingebundenen Mitarbeiters zum Betriebsbeauftragten nachträglich

97 Wenn der Veranlassungsakt ein Verwaltungsakt ist (vgl. §9 VwVfG).

98 Wenn der Veranlassungsakt ein privatrechtsförmiger Vertrag oder ein Verwaltungsvertrag (welcher grundsätzlich ebenfalls dem Kartellvergaberecht unterfällt; vgl. Burgi, Der Verwaltungsvertrag im Vergaberecht, NZBau 2002, S. 57) ist; $\$ 16$ Vergabeverordnung dünte im Hinblick auf Verwaltungsverträge als die gegenüber $\S \S 20,21$ VwVfG speziellere Norm anzusehen sein. Voraussetzung für die Anwendbarkeit des $\$ 16$ Vergabeverordnung ist selbstverständlich die Erfüllung der übrigen tatbestandlichen Voraussetzungen des Kartellvergaberechts (vgl. \$§ 97 f. GWB). Eingehend zu $\$ 16$ jüngst Quilisch/Fietz, Die Voreingenommenheit bei der Vergabe öffentlicher Aufträge, NZBau 2001, S. 540.

99 Die Leistung ist gem. $\S 8 \mathrm{Nr}$. 1 Abs. 1 VOL/A meindeutig und so erschöpfend zu beschreiben, dass alle Bewerber die Beschreibung im gleichen Sinne verstehen müssen und die Angebote miteinander verglichen werden kổnnen«. Die đrei Eignungskriterien des $\S 97$ Abs. 4 Halbs. 1 GWB, danunter das Kriterium der "Zuverlässigkeit« sind hierauf bezogen. Der Vergabesenat des Kammergerichts hat mit Beschluss vom 5.9.2000 (Az.: KartVerg 15/00) entschieden, dass der Begriff der vergaberechtlichen Zuverlässigkeit unter bestimmten Umständen nicht nur negative Verfehlungen, sondern auch die institutionelle Neutralität und Unparteilichkeit erfassen könne. Vgl. zur Bedeutung der Eignungskriterien und der Leistungsbeschreibung in Privatisierungszusammenhängen Burgi, Vergaberechtliche Fragen bei Privatisienungsvorgängen: Das Beispiel Zusammenarbeit mit dem Sicherheitsgewerbe, GewArch. 2001, S. 217 ff. (220 f.). Die Gewährleistung von Neutralität und Objektivität auf der Seite der mit der Vorbereitung staatlicher Entscheidungen betrauten Privaten verlangen auch Hoppe, Rechtsprobleme bei Standortauswahlverfahren für Abfallentsorgungsanlagen durch private Auftragnehmer, DVB1. 1994, S. 255 (261); Trute, DVB1. 1996, S. 961 f.; Hoffmann-Riem, in: Offentliches Recht (Fn. 2), S. 324. 
von der Umweltüberwachungsbehörde beanstandet wird ${ }^{100}$. Bei der Interpretation der entsprechenden Vorschriften ist der Notwendigkeit funktionaler Äquivalenz stärker Rechnung zu tragen, als dies bislang geschieht. Dort, wo Implementationsinstanzen tätig sind, die staatlicher Regulierung in Gestalt einer Akkreditierungspflicht unterworfen sind, müssen Neutralität und Objektivität als Akkreditierungsvoraussetzungen formuliert sein, wie es bei der Akkreditierung von Umweltgutachtern ${ }^{101}$ bzw. von Benannten Stellen im Produktsicherheitsrecht bereits der Fall ist ${ }^{102}$.

\section{Sachaufklärung und Betroffenenbeteiligung}

Auch hier bilden zunächst administrative Kontakte zwischen Staat und privaten Trägern den Ausgangspunkt, indem die privaten Träger im Veranlassungsakt bzw. im Zusammenhang mit der Akkreditierung ${ }^{103}$ zur Weitergabe erlangter Daten an die Verwaltungsstellen verpflichtet werden, um eine optimale Sachaufklärung zu ermöglichen. Im Veranlassungsvertrag bei einer Verwaltungshilfe müssen Klauseln enthalten sein, durch die dem Verwaltungshelfer einerseits Aufklärungsmöglichkeiten gegenüber den betroffenen Dritten eingeräumt werden und er andererseits zur Beteiligung der Betroffenen verpflichtet wird, wenn er vorplanerische Tätigkeiten ausübt ${ }^{104}$. Kommt es bei einer regulierten Selbstregulierung zur Verzahnung mit behördlichen Entscheidungen, kann dies eine Gelegenheit sein, auf eine unzureichende oder unterbliebene Betroffenenbeteiligung zu reagieren. So muss die Gemeinde im Umgang mit Vorhabenträgern, deren in einem Vorhaben- und Erschließungsplan zusammengestellte Vorstellungen in einen vorhabenbezogenen Bebauungsplan aufgenommen werden sollen, darauf achten, dass die die im Falle eines herkömmlichen Bebauungsplanverfahrens anhörungsberechtigten Dritten rechtzeitig zu Wort kommen können ${ }^{105}$. Die gerade in Verfahren dieser Kom-

100 So im Falle des OVG Münster, ZUR 2001, S. 223. Das Gericht stellte fest, dass »weder Betreiber noch Mitglieder der Geschäftsleitung « zum Immissionsschutz- oder Störfallbeauftragten bestellt werden dürften; des weiteren geht es um die Möglichkeit der Bestellung eines »einem Betriebsleiter vorgesetzten Bereichs- und Abteilungsleiters«.

101 Vgl. $\S \S 9$ i.V.m. 5 u. 10 Abs. 1 Ziff. 5 Umweltauditgesetz.

102 Der vorhandene Normenbestand ist allerdings weder systematisch geordnet noch vollständig, wie Röhl (Fn. 46), S. 87 f., veranschaulicht.

103 Entsprechende Pflichten müssen sowohl als Akkreditierungsvoraussetzung ausgestaltet sein als auch im Wege nachträglicher Sanktionen durchsetzbar sein, worauf Laskowski, in: Verantwortungsteilung (Fn. 5), S. 106 f., hingewiesen hat.

104 Zur Betroffenenbeteiligung in Entscheidungsverfahren, die unter smaßgeblicher Beteiligung von Sachverständigen « ablaufen, vgl. bereits Di Fabio, Verwaltungsentscheidung durch externen Sachverstand, VerwArch 81 (1990), S. 214, ferner Ritter, in: Verwaltungsorganisationsrecht (Fn. 8), S. 232.

$105 \mathrm{Vgl}$. Burgi, DV 33 (2000), S. 205, sowie bereits Erbguth/Wagner, Bauplanungsrecht, 3. Aufl. 1998, Rn. 241 ff., und jüngst Grigoleit, Normative Steuenung von kooperativer Planung, DV 33 (2000), S. 79 ff, ferner Faber, Selbstregulierung und Kooperation bei der Bauleitplanung, in: Erbguth/Oebbecke/Rengeling/Schulte (Hrsg.), FS Hoppe, S. 425 (433 f.). 
plexität und Tatsachenverwiesenheit besonders wichtige Betroffenenbeteiligung darf mit der Einschaltung Privater in den Planungsprozess nicht unter den Tisch fallen, eine Erkenntnis, die im Zuge der jahrzehntelangen Bemühungen um die Wahrung der materiellen Standards, insbesondere der Anforderungen des rechtsstaatlichen Abwägungsgebots (im Anschluss an die bekannte Flachglas-Entscheidung ${ }^{106}$ ) vielfach in Vergessenheit geraten ist. Das Zustandekommen einer den Anforderungen des rechtsstaatlichen Abwägungsgebotes entsprechenden Verwaltungsentscheidung hängt nicht zuletzt von der Einbeziehung sämtlicher relevanter Umstände ab, welche sich wiederum zu einem nicht unerheblichen Teil aus den Verlautbarungen der Betroffenen in den verschiedenen Stadien des Planungsprozesses ergeben. Eine weitere Gelegenheit zur Einräumung von Aufklärungsbefugnissen ${ }^{107}$ wie zur Einbeziehung der Betroffenen bietet die Akkreditierung (einschließlich die nachträgliche Überwachung) einer etwaigenfalls eingeschalteten privaten Implementationsinstanz.

Sollten sich die hiernach bestehenden Möglichkeiten der administrativen Regulierung nicht als ausreichend erweisen, wäre die Statuierung von Aufklärungsbefugnissen bzw. von Beteiligungspflichten unmittelbar durch Gesetz in Betracht zu ziehen. Diese kursorischen Hinweise zum Anliegen der Sachaufklärung sollen freilich nicht ohne den Hinweis enden, dass in der Regel schon die Existenz eines Systems der regulierten Selbstregulierung ein Mehr an Information und Problembewusstsein im Interesse der betroffenen öffentlichen Belange verspricht; man denke nur an die Aufklärungsmöglichkeiten, die sich zwangsläufig innerhalb eines Risk-Management-Systems ergeben.

\section{Transparenz, Beteiligtenanhörung und Begründung}

Zunächst finden sich auch in diesem Bereich unmittelbar gesetzlich begründete Verfahrenspflichten. So dienen die Beratungspflichten der Benannten Stellen gegenüber den Produktherstellern ebenso wie die diesbezüglichen Dokumentations- und Begründungspflichten ${ }^{108}$ der Herstellung von Transparenz, während die Anhörung nach Verantwortungsteilung im Bauordnungsrecht dadurch sichergestellt wird, dass teilweise weiterhin die Baubehörde (und nicht der Bauherr), die Angrenzer benachrichtigt (vgl. z.B. $§ 74 \mathrm{BauO}$ NRW); das Umweltauditgesetz verpflichtet den Umweltgutachter unmittelbar

106 BVerwGE 45, 309 f., und hierzu stellv. Brohm (Fn. 33), § 13 Rn. 28.

107 Wie Röhl (Fn. 46), S. 91 f., berichtet, werden die Benannten Stellen im Produktsicherheitsrecht durch die Akkreditierungsstellen dazu verpflichtet, sich in ihren Verträgen mit den Produktherstellern das Recht zur Durchführung angemeldeter wie unangemeldeter Inspektionen zur Überwachung der Hersteller einräumen zu lassen.

108 Näher geschildert bei Röhl (Fn. 46), S. 91 f. 
zur Anfertigung von Zweitschriften und zur Vorhaltung relevanter Unterlagen ( $\$ 15$ Abs. 2 Ziff. 1 u. Ziff. 4). Unter Umständen kann im Falle eines Pflichtenverstoßes administrativ, d. h. mit einer Aufhebung oder nachträglichen Beschränkung der Akkreditierung reagiert werden. Der an dieser Stelle entstehende Eindruck der Zufälligkeit ist nicht unberechtigt und eine wichtige Zukunftsaufgabe besteht darin, den Verfahrenspflichtenkanon bereichsbezogen, d. h. z. B. für das Sicherheitsrecht sämtlicher Produkte, zu vervollständigen $^{109}$.

Jedenfalls bis dahin kommt es auch hier auf die administrative Regulierung an, konkret auf die Auferlegung von Informations- und Publikationspflichten $^{110}$, von Anhörungs- bzw. Geheimhaltungspflichten ${ }^{111}$ sowie von Begründungspflichten im Veranlassungsakt bei der Verwaltungshilfe bzw. im Verzahnungsakt bei einer regulierten Selbstregulierung. Als Beispiel hierfür sei genannt die in $\S 25 \mathrm{a}$ Abs. 1 Ziff. $3 \mathrm{KWG}$ statuierte Aufzeichnungspflicht betreffend die Risk-Management-Aktivitäten als Voraussetzung für die Erteilung der Bankbetriebserlaubnis gemäß $§ 33$ Abs. 1 Satz 1 Ziff. 7 KWG.

\section{Standorte normativer Verfahrens-Regulierung}

Standorte für normative Regulierungsmaßnahmen nach Privatisierung sind bzw. als Standorte kommen in Betracht: das VwVfG (bzw. die Verwaltungsverfahrensgesetze), die jeweiligen Fachgesetze, in denen die verfahrensrechtlichen Bestimmungen mit den an den unterschiedlichen Sachgesetzlichkeiten orientierten materiellen Regelungen verknüpft sind ${ }^{112}$, das jenseits der sog. Schwellenwerte (vgl. $\S 2$ Vergabeverordnung) im GWB geregelte Kartellvergaberecht und schließlich, in einer vierten Gruppe, das allgemeine sowie das Sonder-Privatrecht und das Strafrecht, welches beispielsweise in Gestalt der Korruptionsvorschriften distanzschutzrelevante Aussagen enthält. Das Privatrecht dient in seiner gegenwärtigen Gestalt vor allem der Entfaltung der gesellschaftlichen Handlungsrationalität und stellt einige Basissicherungen zur Verfügung; es sollte nicht zur Heimstatt des Verfahrens-Regulierungsrechts gemacht werden. Daher wird in der vorliegenden Untersuchung auch nicht der Begriff $»$ Privatverfahrensrecht $\ll^{113}$ verwendet.

109 Die detaillierte Analyse der produktsicherheitsrechtlichen Regelungen mündet bei Röhl in das Fazit, das "von den Benannten Stellen anzuwendende Verfahren « sei wnur nudimentär geregelt" (Fn. 46), S. 91.

110 Vgl. Hoffmann-Riem, DVB1. 1996, S. 231.

111 Zum Geheimnisschutz bei der Einschaltung von Sachverständigen vgl. Seidel (Fn. 10), S. 145 ff. m.w.N.

112 Diese beiden Alternativen sind ausführlich gegenübergestellt bei Ziekow (Fn. 12), S. $100 \mathrm{ff}$.

113 Etwa verwendet bei Hoffmann-Riem, DVBl. 1996, S. 231. 
Zurückhaltung sollte gegenüber Vorschlägen geübt werden, eine rechtsgebiets- und damit fachgesetzübergreifende Reglementierung anhand des jeweiligen Tätigkeitsprofils vorzunehmen, und zwar außerhalb des VwVfG, etwa in Gestalt eines »Sachverständigengesetzes $\aleph^{114}$ (entsprechend denkbar wären Zertifizierungs- oder Verfahrensmanagementgesetze). Abgesehen davon, dass hierdurch eine zusätzliche Ebene zwischen dem allgemeinen Verwaltungsrecht und dem besonderen Verwaltungsrecht eingezogen und das Geflecht der Interdependenzen noch undurchdringlicher würde, ist zu bedenken, dass die Tätigkeitsprofile der privaten Träger sich häufig überschneiden. Insgesamt ist bei der Standortdiskussion wiederum zu bedenken, dass die verfahrensbezogene Regulierung nur einen Teil betrifft und gemeinsame Standorte für die verschiedenen Regulierungsgegenstände (die materiellen, die organisatorischen und eben die verfahrensbezogenen) konzeptionell wie verwaltungspraktisch vorzugswürdig erscheinen.

\section{Bei ausschließlich normativer Regulierung}

Normen ohne Bezug zu administrativen Kontakten zwischen dem Staat und privaten Trägern, also außerhalb eines Verfahrensrechtsverhältnisses, gibt es im Bereich der regulierten Selbstregulierung. Durch sie werden Pflichten der die Selbstregulierung tragenden Kräfte sowie von privaten Implementationsinstanzen festgelegt bzw. die Pflichten des Staates in Anbetracht einer Privatisierung verändert. Auch die Einräumung verfahrensmäßiger Befugnisse, etwa im Interesse der Sachaufklärung (vgl. E III 2), gehört hierher. Als Standort jener funktionalen Äquivalente kann nur das Fachgesetz fungieren, in dem das jeweilige Ordnungskonzept entwickelt worden ist.

\section{Bei auch administrativer Regulierung}

Anknüpfend an die oben (B II u. III) geleistete Unterscheidung der "privatisierten Bereiche« und der denkbaren Verfahrensrechtsverhältnisse (E II) ist zunächst das Verhältnis zwischen der staatlichen Verwaltung und dem jeweiligen privaten Träger zu beleuchten. Gleichgültig, ob die administrative Regulierung sich im Veranlassungsakt der Verwaltungshilfe oder im Verzahnungsakt bzw. in der Akkreditierung bei der regulierten Selbstregulierung manifestiert, stets geht es um ein Verwaltungshandeln für das die »normalen« Verfahrensregeln gelten, also in der Regel das $\mathrm{VwVfG}^{115}$. Mit Hilfe der Regeln

114 So die Forderung von Scholz, ZG 2000, S. 221 ff. Freilich gibt es insoweit schon Ansätze, nämlich in Gestalt der Regelungen über die »öffentliche Bestellung «bzw. »amtliche Anerkennung« von Sachverständigen (zusammengestellt bei Seidel [Fn. 10], S. 167 ff.).

115 Teilweise ergänzt durch das Fachrecht, etwa im Hinblick auf den »Durchführungsvertrag " als Verzahnungsakt beim Vorhaben- und ErschlieBungsplan nach § 12 BauGB. 
über die Nebenbestimmungen zu Verwaltungsakten, über die Gestaltung verwaltungsvertraglicher Beziehungen oder - bei Verwirklichung der entsprechenden Vorschläge - die rechtsformunabhängige Gestaltung von Kooperationsbeziehungen ${ }^{116}$ kann und muss insbesondere die Herstellung von Transparenz und Sachaufklärung ${ }^{117}$ zwischen den Partnern bewirkt werden. Zielt die Auswahl des privaten Partners auf den Abschluss eines »entgeltlichen Dienstleistungs-, Bau- oder Liefervertrags mit einem Dritten« ( $\$ 99$ Abs. 1 GWB), was bei der Verwaltungshilfe regelmäßig der Fall ist, unterfällt der Veranlassungsvorgang dem GWB-Vergaberecht ${ }^{118}$.

Hinsichtlich der noch wichtigeren Verfahrensstrukturen im Verhältnis des privaten Trägers zu Dritten, die ja durch den Staat erst initiiert werden müssen (E I), sind bei der Verwaltungshilfe wiederum entweder das VwVfG oder das Privatvertragsrecht relevant (je nach Rechts- und Handlungsform des Veranlassungsaktes; vgl. bereits E III 1 am Beispiel des Distanzschutzes) sowie das GWB-Vergaberecht. Indem dieses die Auswahl des Verwaltungshelfers steuert, ist es zugleich Privatisierungsfolgenrecht und damit unter anderem auch Verfahrens-Regulierungsrecht. Es kann dieser Aufgabe, etwa im Hinblick auf die Gewinnung eines neutralen und verfahrenskompetenten Privaten, zuverlässiger und vorhersehbarer gerecht werden ${ }^{119}$, wenn die entsprechenden Anforderungen im jeweiligen Fachgesetz oder auch in einem künftig das Kooperationsrecht aufnehmenden VwVfG, formuliert sind. Nach Abschluss des Auswahlvorgangs geht es um die Etablierung der erforderlichen Verfahrensstrukturen für den Alltag der Verwaltungshilfe oder, mit anderen Worten, um die »Gestaltung und Begrenzung « der Verwaltungskooperation ${ }^{120}$. Dies geschieht mit Hilfe von Vertragsklauseln, die wiederum in einem reformierten VwVfG gesetzlich determiniert werden könnten. Die diesbezüglichen Vorschläge ${ }^{121}$ sind freilich in verfahrensmäßiger Hinsicht noch ausbaufähig, kon-

116 Notwendigkeit, Möglichkeiten und Grenzen der Schaffung entsprechender Normen sind in den durch das Bundesinnenministerium aufgegebenen Gutachten von Ziekow (Fn. 12), S. 167 ff., und Schuppert (Fn. 12), erörtert.

117 Ziekow (Fn. 12), S. 199, schlägt die Aufnahme eines »§ $55 \ll$ in das VwVfG vor, in dem die "Zusammenarbeit bei der Sachverhaltsermittlung« zwischen den Kooperationspartnern geregelt werden soll.

118 Vgl. nur Jaeger, Vergaberecht und Public Private Partnership, NZBau 2001, S. 1 ff.; Burgi, NVwZ 2001, S. 603 ff. m.w.N. Das Kartellvergaberecht ist nur dann nicht anwendbar, wenn die vom EuGH in seiner Entscheidung zur Rechtssache »Teckal « (EuZW 2000, S. 246, Rz. 49 - 51) geschilderte Ausnahme eingreift, weil der Staat über den privaten Träger veine Kontrolle ausübt wie über . . . eigene Dienststellen und wenn diese Person zugleich ihre Tätigkeit im Wesentlichen« für die staatliche Stelle verrichtet. Zur Einbeziehung auch der Verwaltungsverträge vgl. bereits Fn. 98.

119 Ausführlich hierzu Burgi, GewArch 2001, S. 217 ff., ferner ders., NVwZ 2001, S. 601 ff.

120 Ziekow (Fn. 12), S. 190.

121 Bei Ziekow (Fn. 12), S. 190 ff., und bereits zuvor Bauer, Verwaltungsrechtliche und verwaltungswissenschaftliche Aspekte der Gestaltung von Kooperationsverträgen bei Public Private Partnership, DÖV 1998, S. 89, und ders., in: Schuppert, Verantwortungsteilung (Fn. 5), S. $247 \mathrm{ff}$. Hingewiesen sei ferner auf das von Hillermeier/Castorph/Hartmann hrsg. wHand- 
zentrieren sie sich - naheliegenderweise - doch auf die materiellen Leistungspflichten. In Umsetzung der hier herausgearbeiteten Strukturschaffungspflicht sollten insbesondere Neutralitäts- und Sachaufklärungspflichten, Pflichten zur Betroffenenbeteiligung und zur Beteiligtenanhörung sowie zur Begründung in den entsprechenden Katalogen, u. U. sogar als »Mindestinhaltsklauseln ${ }^{122}$, nicht fehlen.

Infolge der thematischen Heterogenität vorwiegend in die jeweiligen Fachgesetze gehören dagegen die Anforderungen an das Verfahren privater Kräfte und von privaten Implementationsinstanzen bei der regulierten Selbstregulierung. Die - ergänzungsbedürftigen - fachgesetzlichen Akkreditierungsvorschriften sind jedenfalls (auch) Bestandteil des Privatisierungsfolgenrechts und tragen zur Bewirkung funktionaler Verfahrens-Äquivalenz bei.

\section{F. Schluss}

Anknüpfend an die eingangs (A I u. II, B I) formulierte Aufgabenstellung sei die Hoffnung formuliert, dass die Stafette der Dogmatisierungsbemühungen ein Stück weitergetragen werden konnte, wenngleich sie sich angesichts der fortbestehenden Notwendigkeit bereichsspezifischen Analysierens und aufgrund des noch zu verfeinernden bzw. vertiefenden Gesamtsystems der Regulierung noch nicht im Ziel befinden kann.

buch für die Vertragsgestaltung und Sammlung von Vertragsmustern mit Erweiterungen kommunales Vertragsrecht « (seit 1985) und auf das im Jahre 2000 erschienene Handbuch der kommunalen Vertragsgestaltung« von Bergmann/Schumacher (Hrsg.), dessen Band III sich ausschließlich mit der »Privatisierung von Dienstleistungen « beschäftigt, wobei die in beiden Bänden zusammengestellten Vertragsmuster schwerpunktmäßig jeweils den Bereich der Durchführungsprivatisierung betreffen.

122 Die von Ziekow (Fn. I2), S. 192 f, vorgeschlagenen »Mindestinhaltsklauseln « enthalten als bislang einzigstes Verfabrenselement die »Pflicht der Kooperationspartner zur gegenseitigen Information«. 
